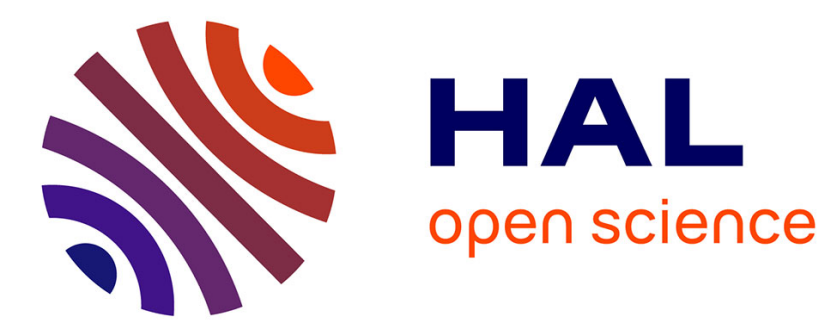

\title{
Un gobelet à décor moulé en sigillée claire $\mathrm{B}$ Colette Bémont
}

\section{To cite this version:}

Colette Bémont. Un gobelet à décor moulé en sigillée claire B. Gallia - Fouilles et monuments archéologiques en France métropolitaine, 1974, 32 (1), pp.83-105. 10.3406/galia.1974.2654 . hal01934650

\section{HAL Id: hal-01934650 \\ https://hal.science/hal-01934650}

Submitted on 25 Feb 2020

HAL is a multi-disciplinary open access archive for the deposit and dissemination of scientific research documents, whether they are published or not. The documents may come from teaching and research institutions in France or abroad, or from public or private research centers.
L'archive ouverte pluridisciplinaire HAL, est destinée au dépôt et à la diffusion de documents scientifiques de niveau recherche, publiés ou non, émanant des établissements d'enseignement et de recherche français ou étrangers, des laboratoires publics ou privés.

\section{(이) $\$$}

Distributed under a Creative Commons Attribution - NonCommercial - NoDerivatives| 4.0 


\title{
UN GOBELET A DÉCOR MOULÉ EN SIGILLÉE CLAIRE B
}

\author{
par Colette BÉMONT
}

Le classement du matériel sigillé de Saint-Rémy-de-Provence m'a conduite, voilà quelques années, à identifier plusieurs fragments inédits de bols décorés, relevant d'une technique assez décadente'. L'étude des tessons, appartenant à la variété dite sigillée claire B - depuis les travaux de N. Lamboglia ${ }^{2}$-, a permis d'établir l'homogénéité globale (malgré des nuances) d'un type de décor à colonnettes, de déterminer un répertoire et de définir, par l'opposition de l'un à l'autre, deux modes décoratifs : l'un se caractérise par son ordonnance et sa régularité rythmique et se fonde sur des principes simples de répétition symétrique; l'autre utilise une série de poinçons partiellement identique dans des compositions de structure plus anarchique ${ }^{3}$.

Depuis cette publication, peu de nouveaux témoins se sont ajoutés à la série : un article récent n'apporte que deux petits fragments d'une frise à grosses colonnes, mais de structure inconnue, qui proviennent de Vaison-la-Romaine ${ }^{4}$. Aussi la découverte de

1 Décors à colonnelles sur des bols de sigillée claire B, dans Gallia, XXVIII, 1970, p. 214-234 (abrégé, par la suite, C.B.'.

2 Vune osservazioni sulla "lerra sigillata chiara" (lipi $A$ e B), dans Revue d'études ligures, $24^{\mathrm{e}}$ an., 1958, p. 257-330. J'ai conservé par commodité cette terminologie. Voir sur le point de ces dénominations l'article de J. et Y. Rigorr et II. Vertet, Essai de classement synthétique des céramiques sigillées, dans Rev. arch. Centre, 45-46, 1973 , p. $69-76$.

3 C.B., p. 216-224. Cies techniques sont illustrées, pour l'une, par un bol de Glanum inv. 1926-1930;, un autre de Vintimille ( $N$. LamboglaA, o. c., p. 321-322) et deux fragments de Valence (G..B., fig. 5, 2-3 et 6, 3-4), pour l'autre par un bol de la collection Gilles (M. 95) conservé à Saint-Rémy-de-Provence.

4 A. Darton, Sigillèe claire $B$ de la vallée du Rhine, dans Revue d'éludes ligures, $38^{\circ}$ an., 1972, p. 181, $n^{\circ} .4$ (inv. 61/116). Je me bornerai à signaler, à titre d'information, un fragment de manche de patère conservé au Musée Réattu d'Arles, décrit et dessiné par J. Déchelette (Vases céramiques ornés de la Gaule Romaine, 1904, II, p. 317 et pl. VIJ, 3! et visiblement apparenté à notre séric. L'objet comporte, in effet, un poinçon absolument identique à notre croix de Saint-André, un fragment d'une colonnette semblable à celle du bol Gilles, le grand chevron, avec la même légère dissymétrie mais comportant, en plus, un point central. L'apparence extérieure du fragment (pâte beige rosé, engobe rougeàtre) ne fournit pas d'argument déterminant en faveur d'un rattachement à la sigillée claire $\mathrm{B}$, sans exclure formellement cette possibilité. Altribuer, comme on l'a fait, le tesson à Lezoux fournirait le premier indice d'un rapport direct entre cet atelier et les decors aux colonnes. I'ne analyse de l'objet permettra peut-être d'en déterminer la provenance, mais, en tout cas, les traditions relatives à celte origine sont assez confuses : Déchelelle, en 1904, le classe parmi des vases provenant "pour la plupart " de la fabrique arverne of signale qu'il est conservé au musée d'Arles. Or la note collée au revers du manche situe le don au musêe (par un collectionneur privé) en 19051906, attribue le fragment à Lezolzx et renvoie a la publication de Jechelette. On peut conclure de cette apparente 


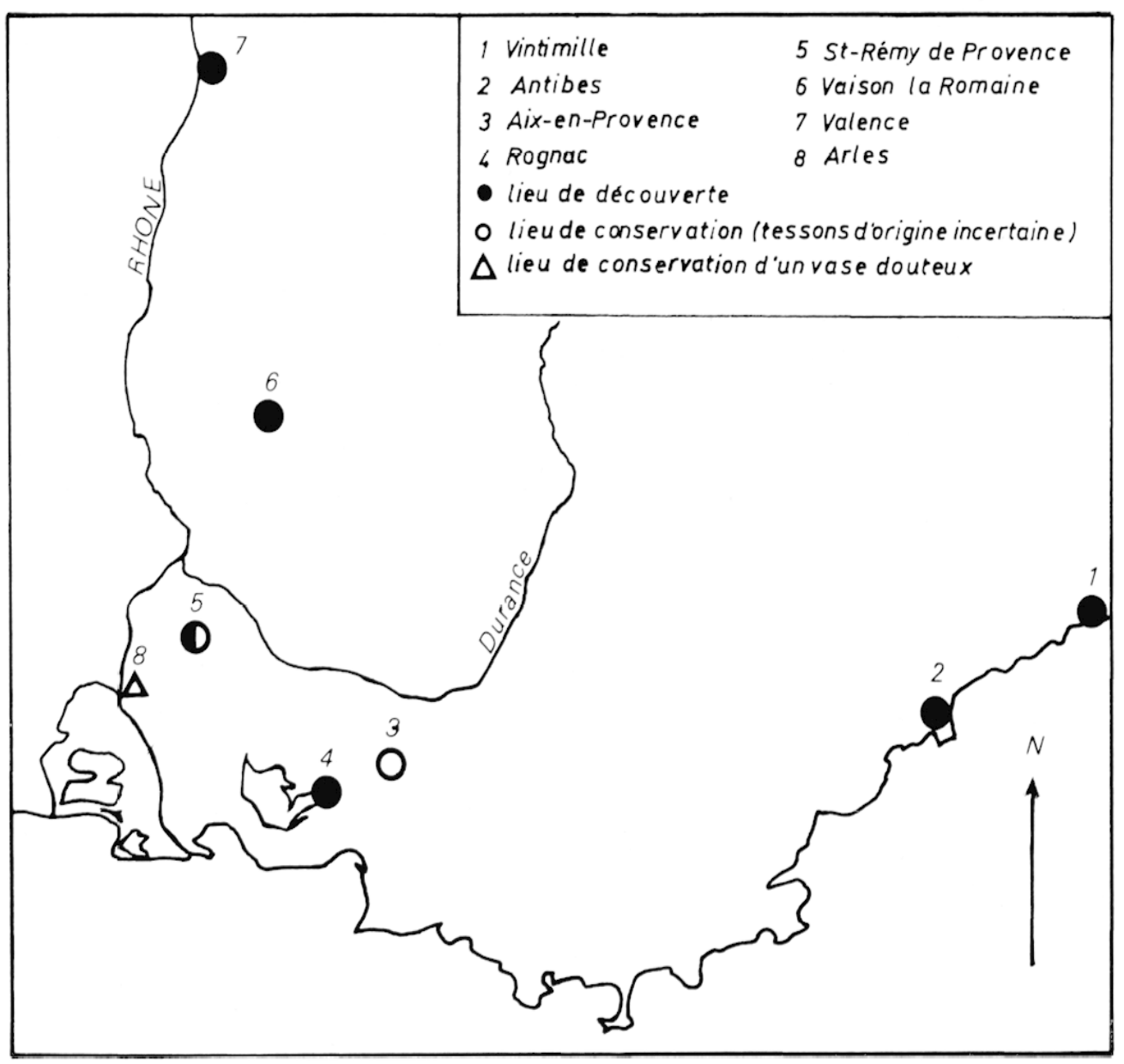

I Répartition des vases a colonnettes.

plusieurs pièces de collection ne saurait-elle laisser indifférent. Encore ne faut-il pas surestimer l'importance de ces trouvailles : il s'agit, en effet, presque toujours, de minces vestiges dépourvus de contexte archéologique et qui permettent, au plus, soit d'enrichir le répertoire décoratif de l'atelier, soit de préciser la carte de répartition des vases (fig. 1). Tel est le cas, du moins, des premiers tessons que j'examinerai rapidement.

Le plus gros fragment, conservé au Musée Granet, à Aix-en-Provence, provient,

contradiction au moins que l'étiquette est postérieure a $1905-1906$ et se demander, en conséquence, si l'altribution à Lezoux qui y figure ne se réfère pas abusivement à l'inventaire, pourtant prudent, de Déchelette, plutiot qu'à une tradition locale relative aux circonstances de l'acquisition. L'examen du décor ne permet pas de résoudre li difficulte puisque, en dehors des motifs déjà signalés, le manche porte une sorte de petite Abondance libant, qui na dite répertoriée ni à Lezoux, ni sur la sigillée claire. On notera, cependant que la juxtaposition des poinçons se fait avec une particuliere maladresse. Il n'est pas exceptionnel, en effet, d'observer sur les pateres de l.ezoux, comme on le voit sur le tesson d'Arles, un léger chevauchement du décor du manche sur le disque terminal ; en revanche, lapplication malencontreuse du motif quadrangulaire de la croix de Saint-André au milieu de l'élégrant ornement curviligne du disque parait étrangère aux habitudes que révèle la série de Lezoux récemment publiée (H. Vertw, Manches de patères ornés en ceramique de Lezoux, dans Ciallia, 30, 1972, pl. 4 a ; 7 b et e ; 8 a et c ; 11 b, d, g ot h : disques cloutés ou nervurés; pl. 5 a et b; 12 a : coquilles). Je remercie J.-y. Roufuette, conservateur du musiere, gràce à qui j'ai pu examiner cette piece. 


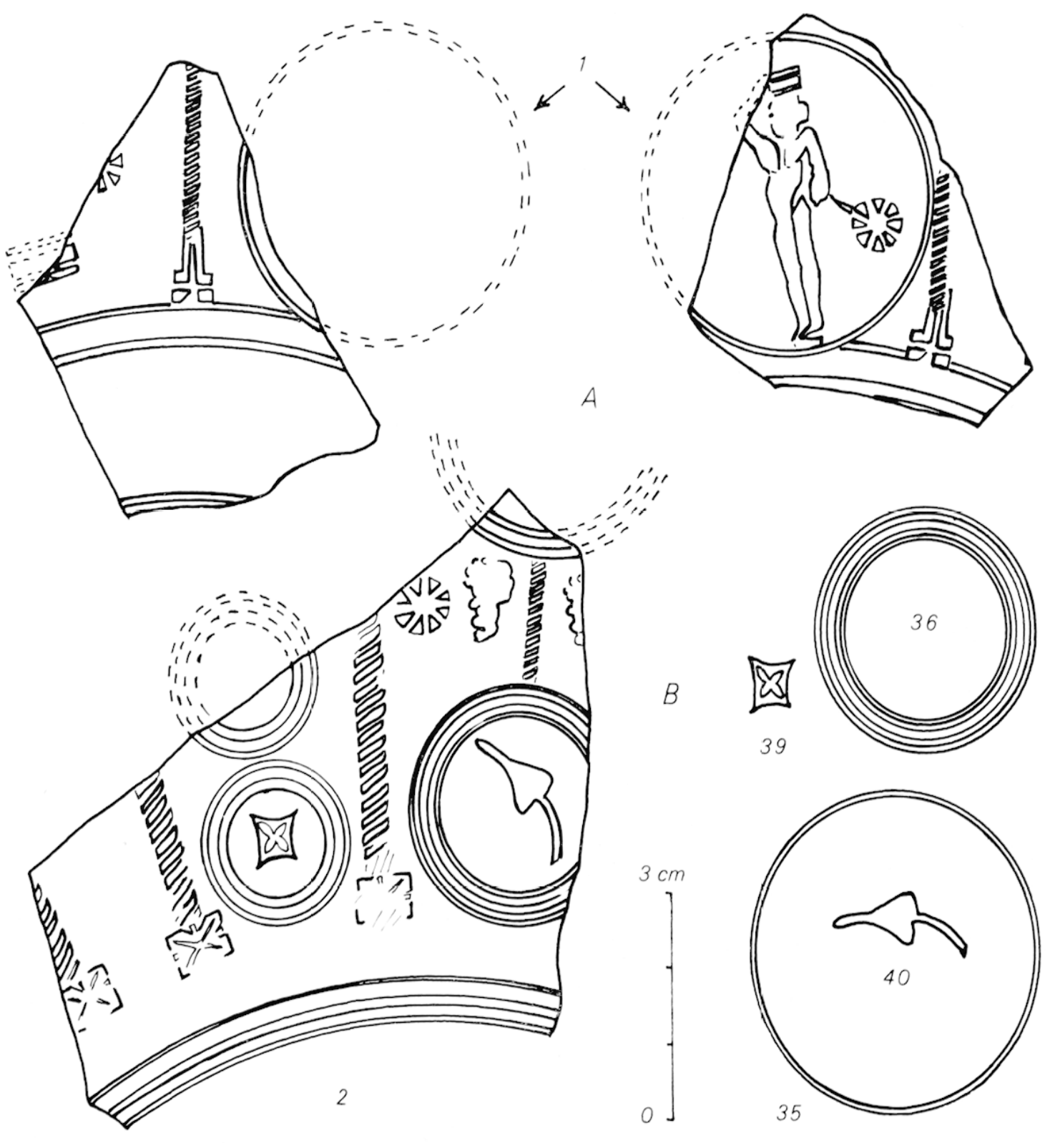

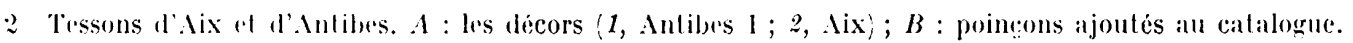

selon toute vraisemblance, d'une petite collection locales. C'est un morceau de bol, dont la pâte - fine, beige rosé, lourde, mais assez bien cuite - est couverte d'un engobe rougeâtre, aux reflets cireux, largement usé sur les reliefs. La frise à métopes (fig. 2, A 2), subsistant sur environ un huitième de sa longueur totale, contient un mélange de motifs déjà recensés (grosse et petite colonnes, croix de Saint-André, grappe, rosette, peut-être double arceau ${ }^{6}$ )

5) Le conservateur du musie .I. Malbos, qui a bien voulu me communiquer le lesson, tire cette présomption dobservations qu'il a faites sur les dépóts anonvmes qui constituent une partie de l'ancien fonds. Je remercie le Dr (r. Simpson qui m'a signalé l'existrnce de ce fragment.

6 C. B., p. 228, $n^{\circ} 5$. L'arc qui subsiste differe par son rayon des petits doubles-cercles, par l'intervalle entre 


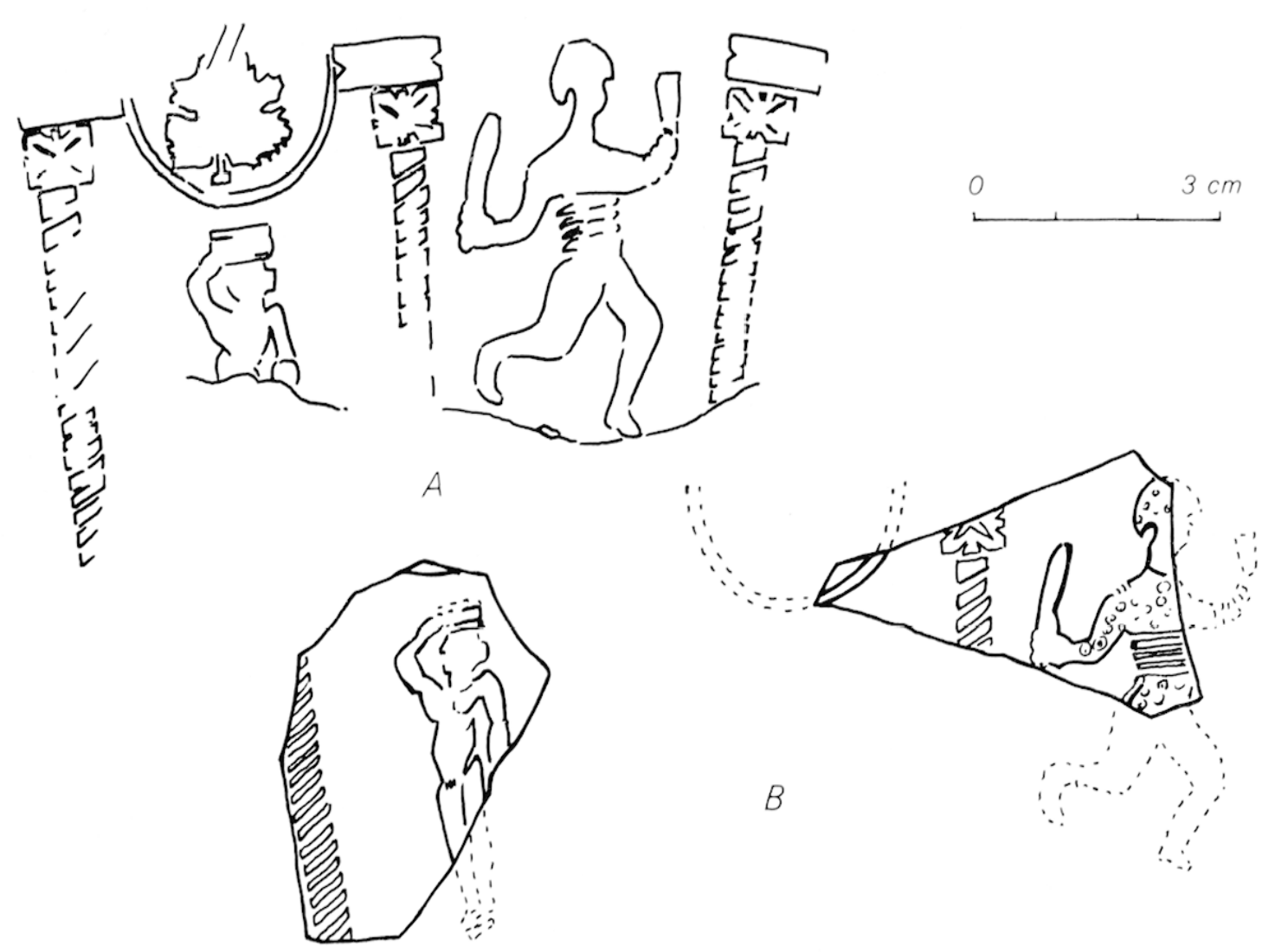

3 Décors au paegniarius. $A$ : fragment du bol 2 de Clanum $: B$ : Lessons d'Antibes 2.

et de poinçons inédits (fcuille d'eau, carré frappé d'une croix, triple cercle) (fig. $2, \mathrm{~B} 36$, 39, 40). L'intérêt de cet échantillon tient, en particulier, à l'usage conjoint de décors attestés, pour les uns, sur des vases à structure régulière et à colonnes épaisses, pour les autres (petite colonne et grappe) sur le bol Gilles?

Quatre autres tessons sont déposés au musée d'Antibes ${ }^{8}$. Ils appartiennent à deux panses de vases. Deux d'entre eux, de provenance locale, ne sont assortis d'aucune référence. Les autres ont été trouvés par G. B. Rogers dans les fouilles de la chapelle SaintEsprit, malheureusement avec un matériel non homogène, dont les éléments les plus tardivement datés sont des minimi de l'époque de Constance II. Les deux vases, semblables par leur pâte fine, beige rosé, rayable à l'ongle, et leur engobe orange vif, solide sur la face extérieure de la paroi, ne fournissent aucun poinçon original : Antibes 2 (fig. 3, l3) se borne à reprendre certains des motifs du bol 2 de Saint-Rémy-de-Provence (paegniarius,

les deux courbes concentriques, du triple-cercle figurant en dessous ; de plus la hauteur déja occupee de la frise et les dimensions habituelles de la colonne rendent improbable, sinon impossible, la présence d'un grand double-cercle a cette place.

7 L'origrinalité du décor lient à l'emploi conjoint des deux motifs de séparation ailleur's exciusifs l'un de: l'aulre; l'un d'eux - la colomette - - est utilisé, pour une fois, comme n'importe quel poinçon décoratif.

8 Je suis reconnaissantr a (i. B. Rogers de me les avoir fail connaître. Les deux vases sont distingues dans la suite de l'exposé comme Antibes 1 ? vase aux colonnettes'et Antibes 2 (vase aux colonnes?. 
canéphore, grosse colonne, arceau, croix de Saint-André) ; Antibes 1 (fig. 2, A 1) associe de façon nouvelle des décors connus : canéphore à la rosette et grand cercle du bol de Vintimille (fig. 2 , B 35) ${ }^{9}$, tau et colonnette du bol Gilles et, probablement, socle triple du bol 2 de Saint-Rémy. L'intérèt de la seconde frisc réside principalement dans l'utilisation, encore inédite, de la petite colonne, comme séparation, dans une construction apparemment régulière et comparable aux compositions à colonnes épaisses.

Tous ces tessons, d'ailleurs, ne conservent la trace que d'organisations à base géométrique et à répétitions, comportant des séparations simples et massives ${ }^{10}$, mais la structure générale des décors nous échappe. Antibes 1, porte en ses parties conservées deux sortes de métopes : les unes avec, les autres sans grand médaillon. Rien n'autorise à supposer que tous les panneaux sans médaillons - incomplets sur les fragments - étaient semblables par leurs proportions et identiquement occupés, ni que tous les cercles contenaient le mème canéphore. L'autre vase est découpé en métopes avec ou sans arceau ; le paegniarius occupe un panneau de la seconde espèce, tandis que le satyre canéphore pourrait se trouver sous un arc. Il est permis d'imaginer, pour les secteurs préservés, une composition proche de celle du bol 2 de Saint-Rémy (fig. 3, A et B) mais les bases de comparaison sont extrêmement réduites et les relevés précis ne permettent pas de superposer les fragments des deux décors assez exactement pour les juger issus du même moule ${ }^{11}$. Le décor d'Aix (fig. 2, A 2), un peu plus étendu, comporte trois panneaux. L'un, très large, en partie détruit, est occupé par un médaillon surmonté d'un secteur de double cercle. Entre ces deux éléments, une colonnette prolongeant le diamètre vertical du médaillon suggère l'existence, à l'intérieur de la métope, d'une symétrie bilatérale : on note, en effet, de part et d'autre de la petite torsade, la présence du même motif, la grappe de raisin. Cette hypothèse, vraisemblable parce qu'étayée aussi par l'existence de structures comparables sur le bol Gilles et sur des fragments de Valence ${ }^{12}$, ne peut être ici absolument confirmée : la partie droite du panneau a disparu. La deuxième métope renferme deux doubles-cercles alignés verticalement; la dernière, la plus étroite, est vide en sa partic basse, seule conservée. Ses dimensions l'apparentent exactement à un panneau de Valence, également libre en bas et occupé, vers le milieu de sa hauteur, par le canéphore, mais cette comparaison, dont la fragilité ne peut échapper, tend surtout à montrer que le caractère exclusivement géométrique des éléments de décor qui subsistent ne saurait impliquer aucune généralisation quant au contenu de l'ensemble de la frise. On remarquera, en tout cas, que la diversité des aménagements préservés exclut la simple alternance de deux métopes et que le bol d'Aix, à cet égard, ressortit à la même série que le bol 2 de Saint-Rémy.

Comme nous le royons, ces modestes fragments ne modifient pas de façon très notable la connaissance de l'atelier aux colonnes. Tout au plus doit-on noter l'usage abondant

9 On parvient à celle conclusion en comparant la taille de ces poincons à celle des autres décors sùrement identifiés de Vintimille.

10 C. B., p. 220-224.

II Peut-être les fragments préservés appartiennent-ils sur l'un à des parties détruites de l'autre; peut-être la qualité du relief - très différente d'un échantillon à l'autre - explique-t-elle ces dissemblances. Toujours est-il qu'on ne peut d'aucune faron superposer les métopes au paegniarius appartenant aux deux bols.

12 C. B., p. 222 , fig. 5,1 et 2. 


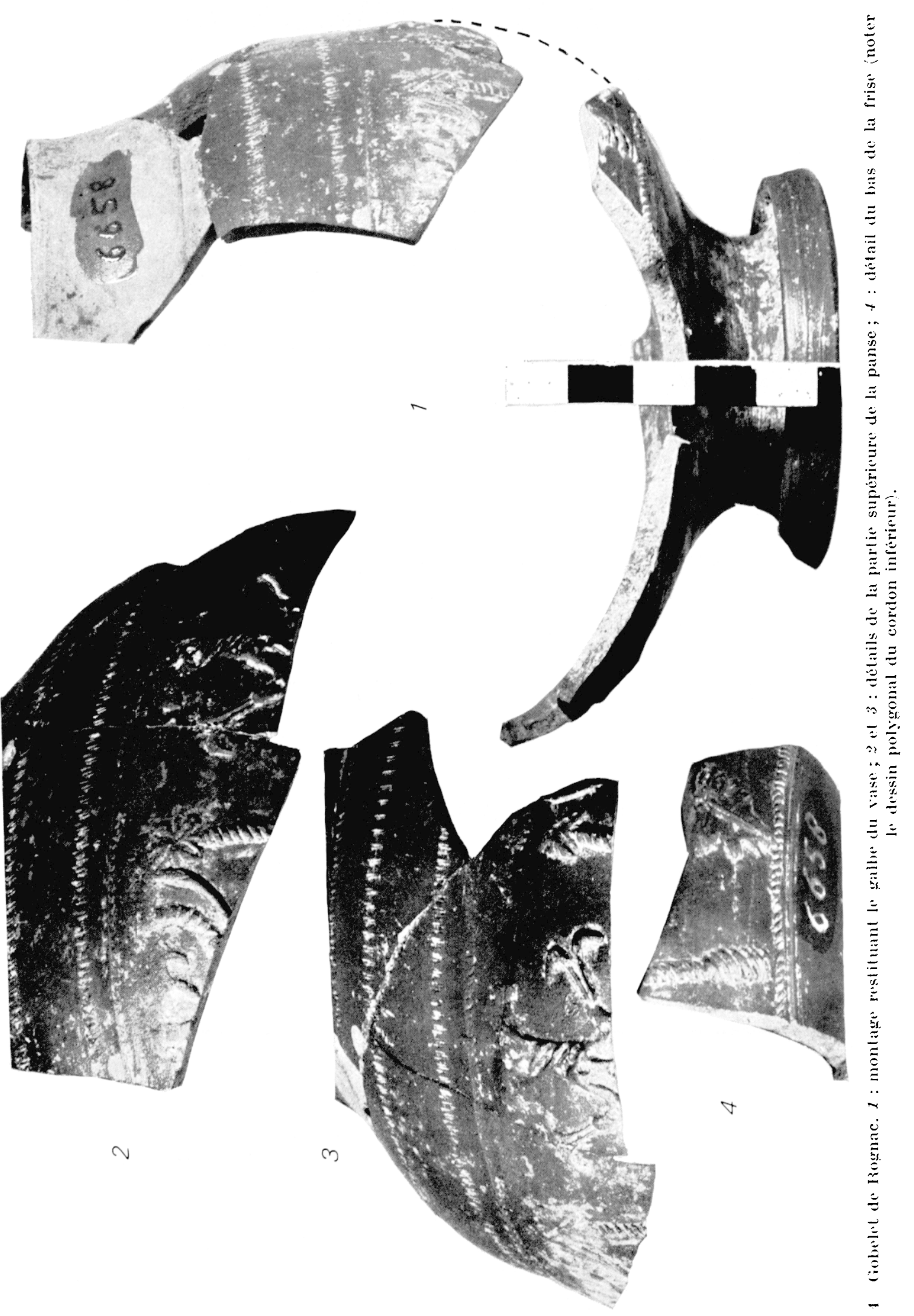


des médaillons qui confirme l'influence probable d'une mode apparue dans la seconde moitié du II $^{\mathrm{e}}$ siècle.

La dernière découverte, en revanche, réservait des surprises. Un groupe de fragments, que j'avais trouvé dans les réserves du Musée Borély, à Mlarseille, me parut devoir être attribué au même décorateur. L'identité presque complète du catalogue des décors avec un ensemble de motifs déjà inventoriés méritait, au plus, d'attirer l'attention sur un nouveau site : Rognac (Bouches-du-Rhône), d'où provenaient les tessons, mais la forme du support se révéla surprenante, au fur et à mesure du remontage et de la restitution graphique de l'objet. Aussi m'a-t-il semblé utile d'analyser plus longuement ce modèle inédit.

Les tessons proviennent des fouilles menées en 1927/1928 par H. de Gérin-Ricard et $P$. Chanfreau dans le quartier de Canourgue ${ }^{13}$ : c'est ce qu'atteste une note manuscrite conservée avec les fragments. Ceux-ci, selon le même rapport, ont été dégagés en même temps qu'un petit autel également déposé au Musée Borély et la célébrité de ce dernier ${ }^{14}$ a permis de contrôler la relation entre texte et céramique et de connaître les circonstances de la découverte du vase. Les inventeurs, en effet, dans un article consacré à l'Autel magique de Rognac, signalent, parmi les matériaux enfouis dans la vase d'une des piscines jumelles, sous la couche de décombres qui contenait un morceau de l'autel, des débris de céramique fine $^{15}:$... « fragments appartenant à trois ou quatre vases dits samiens mais de fabrication locale imitant la céramique de Lezoux ». "Ces fragments", poursuivent les auteurs, "appartiennent à une jolie coupe à pied avec décor en relief et à deux autres vases unis dont un porte au fond et dans un cercle l'empreinte illisible d'un cachet de potier gallo-romain ${ }^{16}$. Nos tessons ne peuvent appartenir qu'à la jolie coupe à pied de fabrication locale. Malheureusement la fouille ne fournit pas de lerminus ante quem pour la destruction et la fabrication de l'objet : Gérin-Ricard et Chanfreau datent, en effet, la ruine des bâtiments, attestée par les décombres, grâce à la céramique, qu'ils situent "entre la moitié du II $^{\mathrm{e}}$ siècle ... et, au plus tard, le milieu du III ${ }^{\mathrm{e}}$ siècle, où son usage disparaît totalement ». Il paraît difficile, dans ces conditions et faute d'autre critère local extrinsèque, de dater la poterie elle-même autrement qu'en se fondant sur des analogies typologiques ou des parentés stylistiques.

Les huit tessons se groupent en deux séries non jointives : l'une couvre le col et le haut de la panse, l'autre comprend le pied et le fond du vase (fig. 4, 1), tout au plus peut-on supposer un point de contact entre les deux morceaux reconstitués. Cependant, un ensemble d'indices incite à penser qu'il s'agit des restes d'une seule et même pièce. Trouvés ensemble, ils présentent, en effet, le même aspect : pâte beige, fine, rayable à l'ongle, mais à cassures

13 F. Brosort, Carle archéologique de la (iaule romaine, fasc. V. Carle (partie occidentale) et lexte complet du départemenl des Bouches-du-Rhône, 1936, p. 89, $n^{\circ} 271$, fournit une bibliographie de ces fouilles. Les tessons, répertoriés au Musée Borély sous le $n^{\circ} 66 \overline{8} 8$ ont été mis à ma disposition par le conservateur, II me S. Bourlard-Collin, à l'amabilité de qui je dois egalement les photos de l'objet.

14 Il s'agit d'un autel magique à inscription grecque (E. Espíraxdec, Inscriptions latines de Gaule (Narbonnaise), Paris, $1929, \mathrm{n}^{\circ} 658$ ). Les deux fragments du petit monument, après la publication du premier découvert, furent rassemblés et commentés ensemble par H. de Gérin-Ricard et P. Chanfreau (L'aulel magique de Rognac, dans Provincia, 1929, p. 31-37). Voir également la chronique archéologique d'A. Grenier (Rev. ét. anc., 1932, p. 291).

15 O. c., p. 35-36.

16 L'autre échantillon de sigillée claire est une petite coupe tronconique, dont seule la partic inférieure est conservée. Je n'ai pu lire la signature du troisième témoin, un fragment d'une forme D.33, en sigillée rouge. 

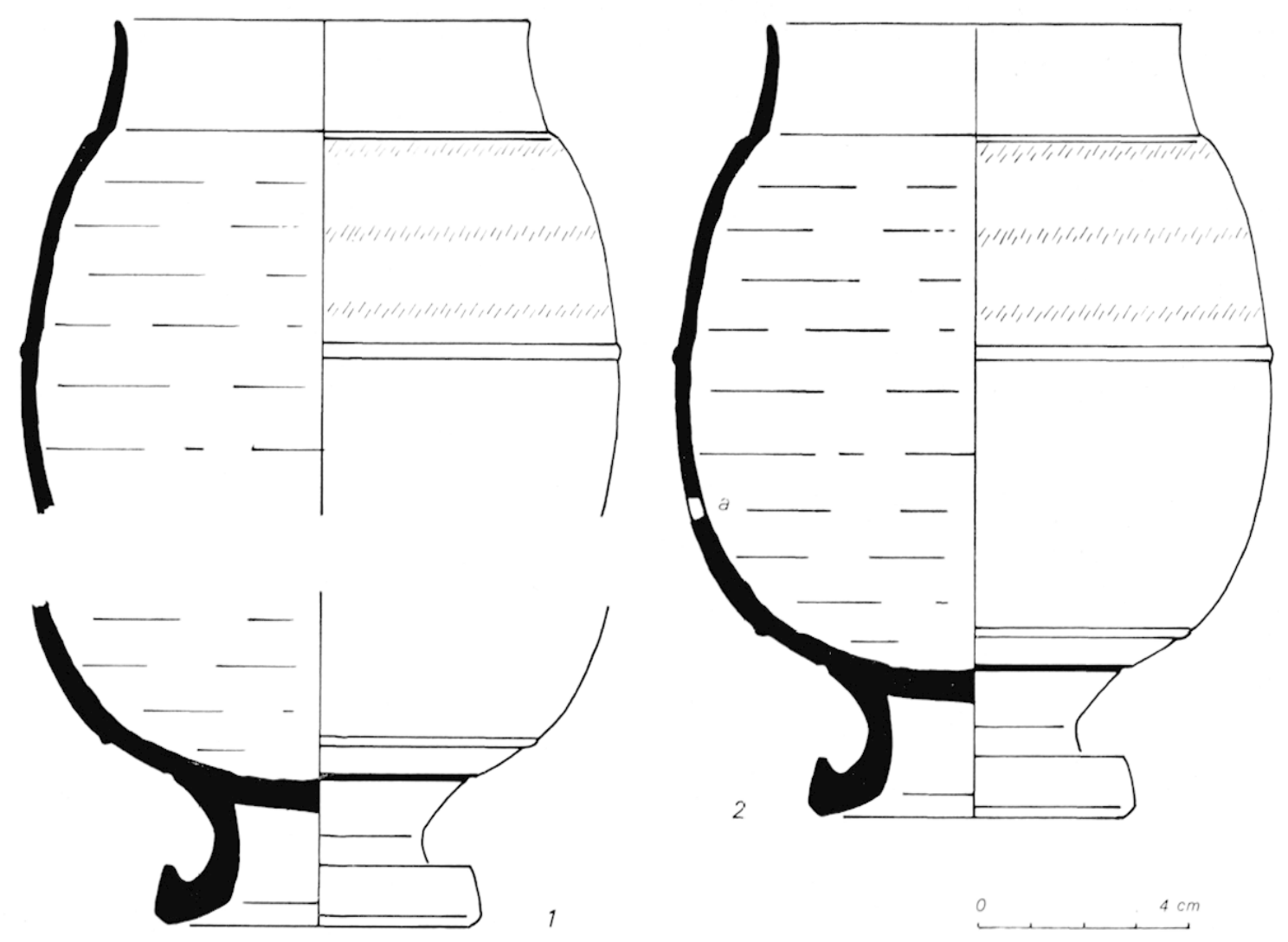

5) Coupe et profil du gobelet. 1 : fragments conservés ; : restitulion graphique (en a zone de superposilion des. relevés parliels.

franches; couverte d'un orangé soutenu, uniforme à l'extérieur ${ }^{17}$, moins régulièrement épaisse à l'intéricur, brillante, mais non métallescente. Par ailleurs, l'identité de courbure, de diamètre, d'épaisseur des parois, permet de raccorder - - au prix d'une superposition de quelques millimètres - - les profils supérieur et inférieur relevés, chacun, sur sa plus grandle hauteur (fig. э). De plus, le décor des deux fragments procède du même répertoire. Autres arguments, négatifs cette fois : la surface interne de la paroi - couverte d'un engobe et marquée uniformément de profondes traces de travail au tour - - exclut que le piédestal ait supporté un vaisseau très étranglé en sa partie supérieure (les cruches ne sont pas, d'ordinaire, engobées intérieurement, ou tout au plus le sont-elles le long du col), ou, au contraire, un calice (forme soigneusement lissée à l'intérieur). Ce sont les vases ovoïdes qui, dans les séries sigillées ${ }^{18}$, réunissent souvent ces deux particularités de finition ; or, ici, le fragment supérieur appartient incontestablement à une forme globuleuse. Enfin, la nature du décor, - divisé par des motifs verticaux, les colonnes - et la technique de moulage employée pour l'obtenir rendent peu vraisemblable l'hypothèse d'un étran-

17 Malgré l'apparence flatteuse, l'adhérence du film n'est pas parfaite : l'un des fragments du bord, comme on le voit sur la pholo, a totalement perdu son engobe extérieur, durant l'enfouissement, ou au cours du nettoyage.

18 J'ai observé ces caractéristiqques particulierement sur des ovoìdes rouges, lisses ou décorés, de la Graufrsenque el sur des ovoïdes moulés, noirs ou rouges, de Lezoux. 
glement de la panse et, par conséquent, d'une solution de continuité entre les deux parties conservées du profil : le rétrécissement du diamètre en bas du fragment supérieur ne paraît donc pouvoir être que la suite naturelle de la courbure du fond.

Le vase, tel que le montre la restitution griphique, est un gobelet trapu, à panse renflée, pourvu d'un col assez court, tronconique, légèrement concave et terminé par une lèvre effilée. La partic inférieure du vaisseau, la plus haute et la plus ventrue, a été obtenue par moulage à l'aide d'une petite forme hémisphérique; une moulure peu saillante indique la limite de la partie supérieure, moins galbée. Hontée au tour, cette dernière est ornée de trois rangs de guillochis (figr. 4, 2 et 3), le dernier se trouvant à la hauteur du léger épaulement qui marque le raceord avec le col. Le tout repose sur un pied creux et proportionnellement élevé, ajouté après le démoulage. De profil fortement concave, celui-ci s'élargit vers la base, en se retroussant vers l'extérieur.

Du point de vue technique, la fabrication de cette pièce s'apparente sans conteste à celle des vases ovoüles produits, en particulier à la (iraufesenque, au ${ }^{\text {er }}$ siècle, et, durant à peu près tout le $\mathrm{II}^{\mathrm{e}}$ siècle à Lezoux ${ }^{19}$ : mème méthode pour obtenir le décor, même association que sur des modeles du Centre de deux modes d'ornementation, le relief et le guillochage. La combinaison mème des différents éléments propres à cette forme se retrouve dans l'élaboration de sigillées classiques. En effet, un type rare de la Graufesenque ${ }^{20}$ réunit de la même façon un pied élevé rapporté, un vaisseau globuleux bipartite, orné, en sa moitié inféricure, d'un décor moulé, et un petit col (fig. 6, 2). Toutefois, malgré d'indiscutables analogies, d'ordre technologique, la distance est considérable entre les deux modèles : on situe les vases de la Graufesenque entre 40 et $70^{21}$ et la production de la sigillée claire $B$ est tenue pour beaucoup plus tardive ${ }^{22}$. D'autre part, le galbe des deux échantillons se ressent notablement de cet écart : le type de la Graufesenque est sphérique et s'achève par un très petit col étranglé, le vase de Rognac est ovoïde, avec un col tronconique à peine concave (fig. 6, 1 et 2 ). Lin fait, son allure générale l'apparente à des formes des $\mathrm{II}^{\mathrm{e}}, \mathrm{III}^{\mathrm{e}}$ et $\mathrm{I}^{\mathrm{e}}$ siècles, bien que les ressemblances avec tel ou tel modèle demeurent très partielles. Yous ne noterons que pour mémoire les petits gobelets de Banassac. Datés actuellement entre 7.) at 125), durée admise de la vie de ce centre, ils pourraient avoir subsisté plus longtemps, si l'on rectifiait la datation générale de la fabrique rutène ${ }^{23}$. Ils

19 Classécs, sous les n $n^{\text {os }} 6$; a $6 x$ par J. Déchelelte, ces formes, assez peu étudiées, me paraissent avoir connu un succes plus vif qu'on ne le montre généralement dans l'entourage du polier PATERNVS, c'est-à-dire vers le milieu du $11^{\mathrm{e}}$ siecle, ou plus tard. Des vases s(mblahles, noirs pour la plupart, ont egalement eté fabriqués à Luxeuil (I. Lerat et Y. Jeaxixix, La céramique sigillée de Laxeuil (Annales lilleraires de l'Lniversilé de Besançon, vol. 31), Paris, 1960, p. $16 ; 65, n^{\circ} 143 ; 7 x, n^{\circ s} 219-223$. I)es moules comparables a ceux de Lezoux sont signalés à Sinzig (Ch. FischLr, Die Terra-sigillala Manufalitur von Sinzig-am-Rhein (Rheinische Ausyrabungen, l. j), 1969, fig. 14 et

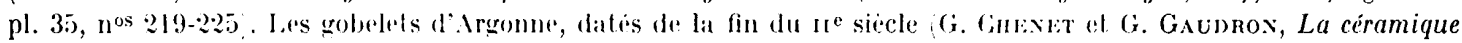

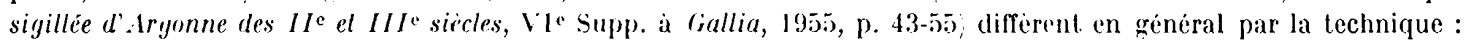
le moule est presque toujours hivalve.

20 F. II:нмет, La Graufesenque, Paris, 1934, vol. II, pl. 1.

21 A. Vernhet, qui a confirmé cette datation, a eu l'amabilité de me signaler des cxemplaires inédits à Bram Audej et Narbonne.

22 La chronologie actuellement admise situe la production entre la fin du I $^{\mathrm{e}}$ siecle et un moment indéterminé du we siecle (N. I.A.mogra, o. c., p. 3:29-330 et Punli fermi sul teatro romano di Vintimiglia, ibid., 1962, p. 273).

23 On tend a prolonger lactivite de Banassac vers la fin du ne siecle en se fondant sur les ressemblances stylistiques decors à arceaux et à médaillons, el sur la communauté de répertoire qui unissent certains décorateurs gabales 

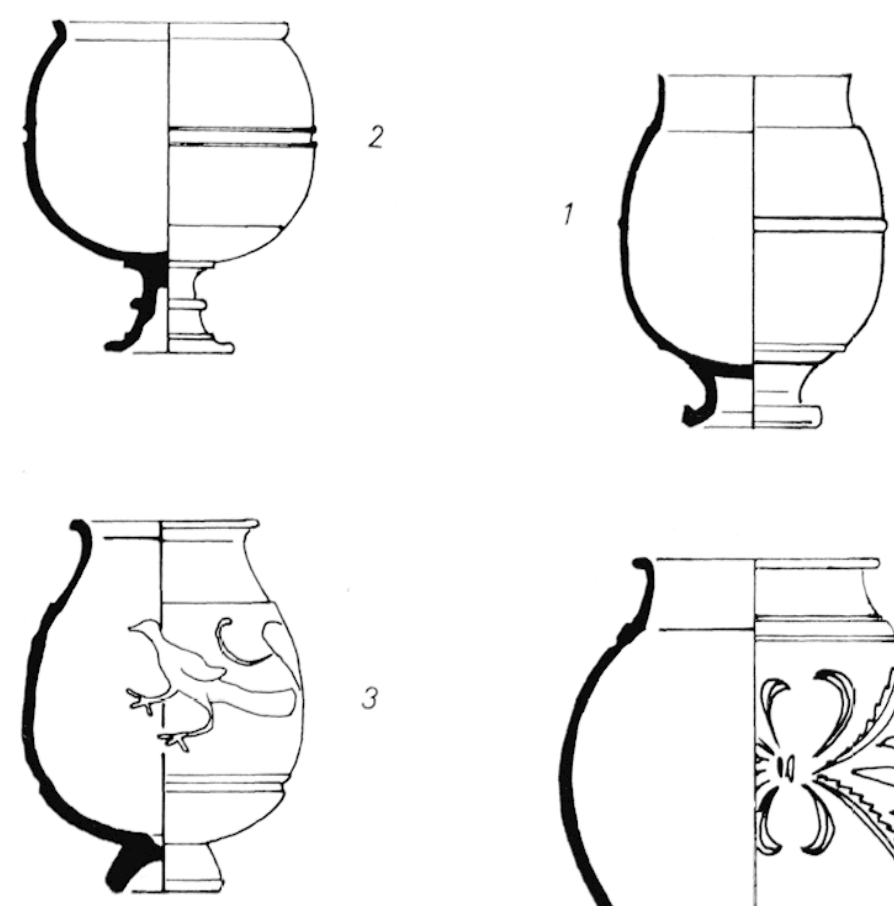

3
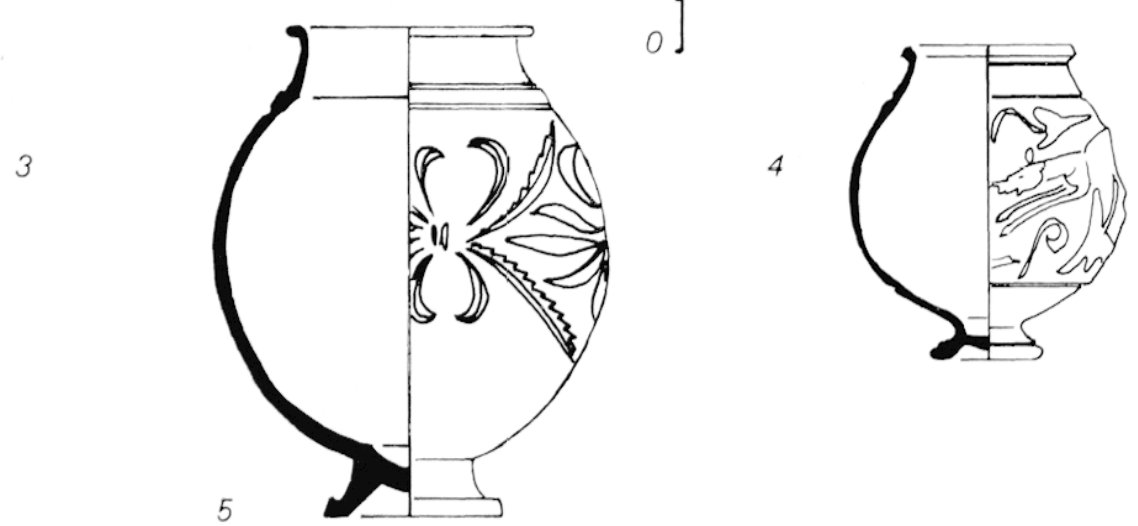

6 Le vase de Rognac comparé à divers échantillons. 1 : vase de Rogniac ; 2 : vase de la Graufesenque d'apres F. Hermet, l. c.) ; 3 : vase de Rheinzabern (d'apres W. Ludowici, Katalog $V, \ldots, 1927$, p. 280, $\mathrm{V} .11 \mathrm{~g}$; ; 4 : vase de Niederbieber (d'apres F. Oelmann, Die Keramili des Kastells Niederbieher, Frankfurt/Nain, 1934, pl. I, 24 c); 5 : vase de Lavoye du In $^{\mathrm{e}} \mathrm{s}$. (d'après G. Chenet et G. Gaudron, l. c.); 6 : vase de sigillée claire $\mathrm{B}$ (d'après N. Lamboglia, l.c.).

comportent effectivement un vaisseau globalement ovoïde et un col tronconique, parfois une sorte de piédestal, mais malgré la similitude d'allure ils diffèrent notablement de. l'excmplaire de Rognac par la technologie - ils sont lisses et entièrement montés au tour ${ }^{24}$ et par le détail du profil : la panse, en effet, s'étrangle en son milieu. De plus, la présence du piédouche est loin de constituer un caractère constant. Ce sont les vases sombres ou rouges du nord-est de la Gaule (Rhin et Argonne), ceux de Castor ${ }^{25}$, placés, pour les plus semblables, au III $^{\mathrm{e}}$ siècle, qui présentent avec notre gobelet les analogies de galbe les moins douteuses : panse globuleuse ou ovoïde, pied étranglé et dégagé, col tronconique, marqué, à son att,ache, d'un épaulement (fig. 6,3 et 4 ). N. Lamboglia a souligné déjà les rapports

et des fabricants de Lezoux, comme CINNAMVS (cf. G. B. Rogers, Banassac and CINXAMVS, dans Rei Crelariae Romanae Fautorum Acta XI/XII, 1969/1970, p. 98-106 ; P. PEYre, Banassac el Lezoux, Centre de Documentation archéologique de la Lozère, 1973,23 p.).

24 La panse est souvent ornée de lignes de guillochis (cf., par exemple, M.A... 19688'.

25 Voir pour le Rhin W. L.cnovici, Kalalog V, Stempelnamen und Bilder Römischer Töpfer, Legions-ziegelslempel, Formen von Sigillala und anderen Gefässen aus meinen Ausgrabungen in Rheinzabern, 1901-1914, 1927, p. 280, V Mg; F. OrLmaxx, Die Keramili des Kaslells Niederbieber (.Mal. zur röm.-german. Keramik, I), Frankfurt/Main, 1934, pl. I, $24 \mathrm{c}$ et p. 31-33. Voir pour la Grande-Bretagne J. P. Gillam, Types of roman Coarse Pollery Vessels in Northern Britain, Newcastle, $3^{\mathrm{e}}$ éd., 1970 , p. 9 el p. $47, \mathrm{n}^{\circ} 50$ (daté entre 200 et 260 ). 
apparents entre la vaisselle claire B et certains modèles de la vallée du Rhin et la similitude est tout à fait évidente pour la forme globuleuse, tournée et ornée d'excisions, qu'il prend comme exemple $e^{26}$ (fig. 6, 4 et 6). Dans la circonstance présente, une particularité du modèle : le profil du pied, suggère des comparaisons surtout avec les produits des ateliers d'Argonne ${ }^{27}$. La base de ces gobelets, en effet, comporte très souvent, au rve siècle, un bourrelet plat, à pente plus ou moins oblique, dégagé sur le dessus par une petite gorge ${ }^{28}$. Toutefois, ces pieds en forme de tampon ${ }^{29}$ sont fermés en dessous et ils représentent l'aboutissement d'une évolution amorcée au III $^{\text {e }}$ siècle avec des échantillons à bourrelet plat et gorge, mais largement évidés en dessous, ainsi que le prouve un vase trouvé dans le four I) de Lavoye $^{30}$ (fig. 6, 5). Aussi, sans négliger la fréquence beaucoup plus grande de ce type de pied au IVe siècle, ni les contraintes imposées peut-ètre à notre potier par le montage d'un piédouche rapporté, estimé-je actuellement prématuré de contester l'importance typologique et chronologique de l'évidement du pied et d'attribuer avec certitude au Iv ${ }^{\mathrm{e}}$ plutôt qu'au in ${ }^{\mathrm{e}}$ siècle le galbe du gobelet de Rognac.

Tel qu'il se présente, en tout cas, cet échantillon se distingue de ses analogues par l'effilement de la lèvre - alors que la plupart des exemples classiques comportent un bourrelet plus ou moins développé - - et par la qualité du décor. Les gobelets de cette époque, en effet, sont d'ordinaire ornés de motifs excisés ou dessinés à la barbotine. Aussi le problème n'est-il pas tellement de savoir pourquoi, par son profil, le gobelet de Rognac ressortit à une typologie commune de la fin du $\mathrm{II}^{\mathrm{e}}$ au $\mathrm{IV}^{\mathrm{e}}$ siècle. La difficulté est plutôt, une fois constaté l'aspect tardif du profil, d'expliquer la résurgence, ou de déceler les traces de continuité d'une technologie connue par des modèles antérieurs de plusieurs décennies, voire de plus d'un siècle. Or les ateliers actuellement publiés ne semblent pas fournir de témoignages en faveur d'une telle pérennité : le récent essai de typologie de Lezoux est muet sur ce point ${ }^{31}$ et on ne signale ni à Sinzig, ni dans les premiers ateliers de Trèves, ni à Rheinzabern, pas plus qu'aux Martres-de-Veyre, chez Satto et Saturninus, ou à Luxeuil ${ }^{32}$, rien de comparable. Quant à la Graufesenque, les travaux de ces dernières années n'ont pas révélé l'existence de proches descendants du type H. $12^{33}$. Force est donc,

26 o. c., p. 312-314, type 28.

27 Je remercie vivement Mme E. Eltlinger des précieuse's suggestions qu'elle m'a faites à l'occasion de cette enquête. Par ailleurs les relations typologiques entre les produits d'Argonne et la sigillée "provencale " ont été soulignées par X. Lamboglia surtout pour une céramique qui pourrait être soit le résultat d'une évolution technique de la sigillèe claire B, soit une production concurrente et similaire parfois exclusive de la claire B, parfois associée à elle) : la céramique luisante „o. c., 1958, p. 298 el Nuove osservazioni sulla "lerra sigillala chiara" (II), ibid., 29c an., 1963, p. $166-168$;.

28 G. Chexet, La céramique gallo-romaine d'Argonne du IVe siècle, Iácon, 1941, pl. XVII-XVIII, type 335 et fig. $33(=$ M.A.N. 28679 et 15014$)$.

29 Cif. ibid., l'analyse de la p. 85.

30 G. Chexet et G. Gavbrox, o. c., fig. 9, C. Le four $\mathrm{D}$ a été utilisé à la fin du $\mathrm{I}^{\mathrm{e}}$ et dans le cours du $\mathrm{II}^{\mathrm{e}}$ siecle (ibid., p. 18; avant 270/275 selon toute vaisemblance. A propos de la mise en sommeil et de la résurrection de l'industrie d'Argonne au we siecle, voir G. Cinsext, o. c., p. 6-7.

31 II. Ventet, Projel d'un répertoire des vases à décor moulé fabriqués à Lezoux, dans Rev. arch. Cenlre, 43-44, 1972 , p. $282-297$.

32 Lorsque la fabrication persiste au u $^{\mathrm{e}}$ siècle, la technique du moulage est remplacee, pour ces formes en tout cas, par la barbotine ou l'excision. E,t, jusqu'à présent, les vases moulés attribués au we siécle de Lezoux ne semblent pas appartenir à ces types (Informations de Gallia, XXVII, 1969, p. 337 ; XXIX, 1971, p. 329).

33 Cetle information me vient d'A. Vernhet. 

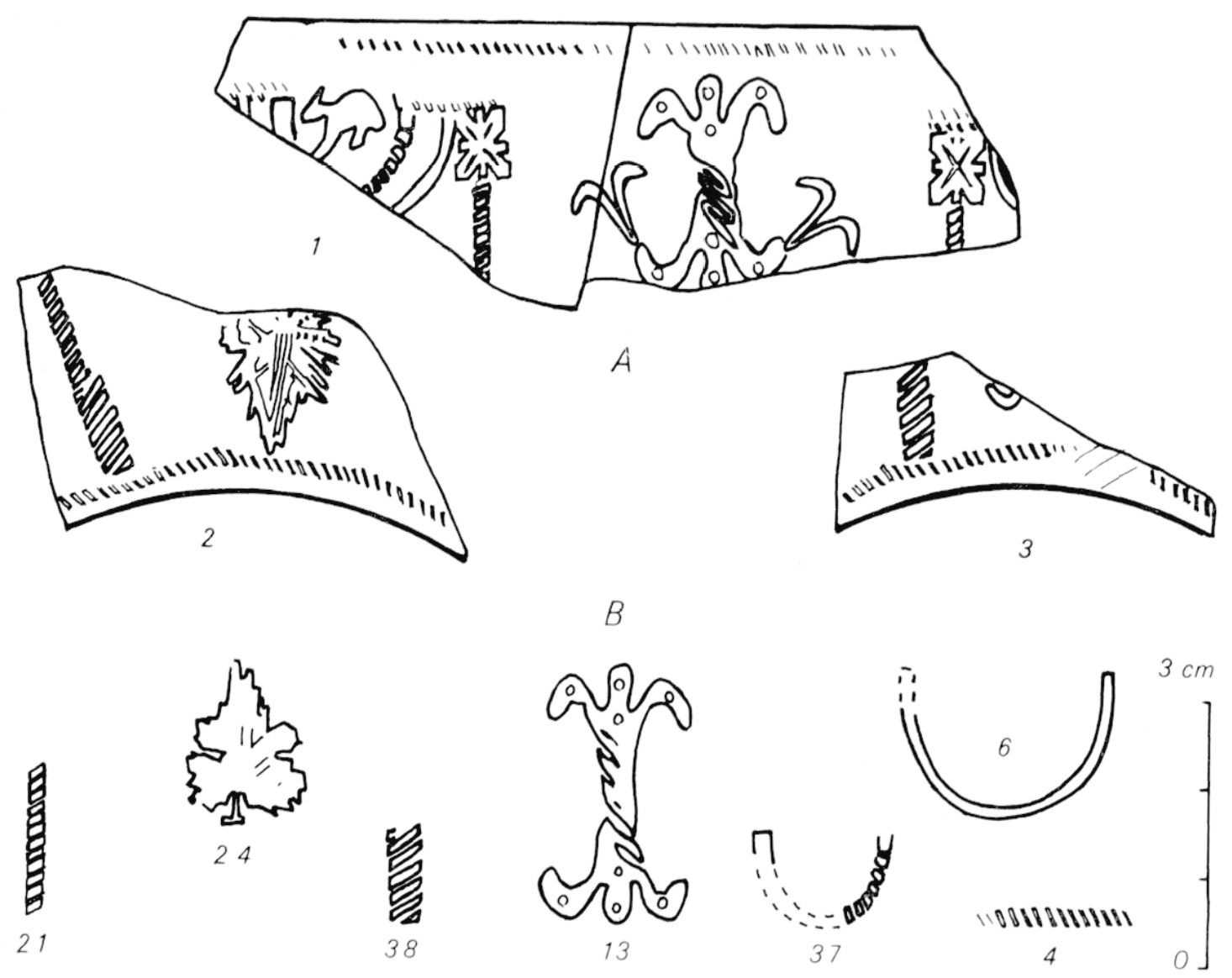

7 Décor du gobelet. $A$ : fragments conservés 1 : partie supérieure; 2 et $3:$ fragrments attenant an pied ; $B$ : poincons (4-24: état le plus complet de poincons ici mutilés; 37 el 38 : nouveaux poincons!.

pour le moment, de constater cette éclipse : il n'est pas possible, en effet, de vicillir l'objet en se fondant sur les seules ressemblances technologiques et en négligeant à la fois les particularités de la forme et la qualité de la céramique.

Le décor du vase (fig. 7), pour sa part, s'intègre aisément dans une série connue, dont on a trouvé des témoins, sur des formes D. 37, à l'est du Rhône, le long du littoral méditerranéen et dans la vallée du fleuve. Le répertoire comprend huit poincons déjà sùrement ou très probablement inventoriés : le cordon torsadé supérieur, la croix de SaintAndré, l'arceau lisse, le grand double-fleuron, le chevron, la colonne, le lapin, la feuille ${ }^{34}$. Deux d'entre eux appellent une remarque. La colonne est semblable à celle du bol Gilles, mais légèrement plus épaisse. Cependant la diversité des deux motifs n'est pas telle que l'on puisse, sur cette seule apparence supposer deux poinçons différents : une impression plus ou moins profonde semble suffire à justifier de faibles variations, que l'on peut, d'ailleurs, observer sur un mème échantillon ${ }^{35}$. D'autre part, le double-fleuron, qui n'était connu

34 C. B., fig. 7, 4, 20,6,13,15, 21, 27, 24.

35 Cf. le bol Gilles. La présence d'une base quadrangulaire sous la colonnette de ce vase ne constitue pas un obstacle à l'identification. Les deux motifs n'avaient chcore été trouvés qu'une fois et associés, mais on pouvait faire 
en son entier qu'à un petit nombre d'exemplaires ${ }^{36}$, est ici raccourci (fig. 7, A 1 et B 13). Il ne s'agit, en effet, ni d'une diminution générale de la taille, qui aurait affecté également la largeur, ni d'un autre poinçon : l'examen attentif du décor révèle un enchevêtrement dans la torsade du centre du motif (ligr. 4,3 et $7, \Lambda 1$ ). On a visiblement, pour gagner de la place, imprimé successivement les deux extrémités du fleuron, en les rapprochant, ce qui entraîne une superposition au milieu ${ }^{37}$. Deux autres poinçons : l'arceau strié et le gros tortillon servant de base à la colonne, n'avaient pas encore été recensés (fig. 7 , B 37 et $38 ; 4,2$ et 4 ). Le premier n'etait pas attesté, le second figure sur un bol de Vintimille ${ }^{38}$, mais n'a pas été publié dans l'inventaire de X. Lamboglia. Deux éléments du décor, enfin, posent un problème. Le motif horizontal unissant les arceaux aux chapiteaux a été écrasé partout où il subsiste (fig. $7, \Lambda$ 1). Les traces de torsades qui restent n'interdisent pas de penser qu'il puisse s'agir du tortillon déjà connu, rien n'exclut, non plus, l'hypothèse de l'emploi d'une matrice encore inédite et inconnue dans son entier. D'autre part, la frise est soulignée par un cordon torsadé. Or ce dernier se présente non pas comme un cercle mais comme un polygone (fig. 4,$4 ; 7, \mathrm{~A}_{2}$ ) et tout invite à penser qu'il a été obtenu par l'impression répétée d'un poinçon rectiligne : une mince torsade d'environ $20 \mathrm{~mm}$ de long. Cependant les différences de pression ${ }^{39}$, dues sans doute en partie à l'inadaptation de la courbure de l'outil au galbe du moule, font varier l'épaisseur du motif et ne permettent pas de savoir si l'instrument utilisé était sùrement le même pour ce cordon et pour la colonnette.

Comme on le constate d'après cet inventaire, le répertoire décoratif est très pauvre et réduit presque exclusivement à des figures géométriques ou architecturales. La vue des tessons est plus décevante encore (fig. 4 et $7, \mathrm{~A}$ ); il ne reste qu'un morceau de la partie supérieure de la zone ornée et, solidaires du pied, deux menus fragments de la même frise. Cependant l'examen de la structure des parties conservées conduit à caractériser un peu plus exactement la composition. Il s'agit ici apparemment d'une construction dont l'esprit rappelle les bols de Glanum, de Valence, de Vintimille : elle se fonde sur l'emploi, en larges ensembles géométriques, de colonnes appuyees sur une base simple et sommées d'un chapiteau, et qui occupent loute la hauteur de la frise ${ }^{40}$. Une différence, toutefois : le choix du fùt torsadé - non pas épais, comme sur les trois vases déjà cités, mais mince, comme sur le bol (Gilles et les tessons d'Antibes 1. Par ailleurs, si la base du décor ne conserve que la trace de deux colonnes disposées selon un plan incompréhensible au premier examen, le fragment supérieur -- correspondant à un peu plus du quart de la circonférence de

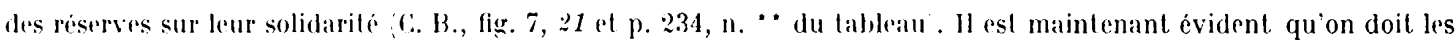
séparer cf. infra. p. 100. Les fùts torsadés sont sensiblement de la même hauteur sur be bol et sur le gobelet, autant que permette d'en juger l'impression, aux extremités de ceux-ci, des bases el des chapiteaux.

36 C. B., fig. 1 in 3.

37 L'etal des exemplaires conservés assez largement (trois à Glanum el celui de liognac: ne permet pas de voir si fe double-fleuron a éte obteru jar l'impression - tantot unique, tantót en deux trmps - d'un seul poinçon comportant l'ensemble du motif, ou si l'on a utilssé, pour chaque decor, un fleuron simple imprimé deux fois.

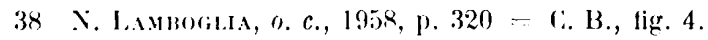

39 Le's angles du polygone presentent des épaississements dus peut-être aussi à la superposition des motifs, a lendroit de leur jonction.

40 Le bol Ciilles, en revanche, est orné de minces colonnetles reposant sur trois éléments de base disparates et superposis, destines apparemment à compenser te défaut de hauteur du fùt par rapport à la zone ornée.
} 
la panse - témoigne sans doute possible d'une organisation symétrique (fig. 7, A 1) : en effet, la symétrie autour d'un axe vertical, propre au double-fleuron, est soulignée par deux chevrons disposés semblablement, de part et d'autre du motif, et le groupe, lui-même, est placé exartement au milieu d'un panneau ${ }^{41}$ qu'encadrent deux colonnes soutenant chacune un arceau simple. Le diamètre de ce dernier, conservé à gauche du fragment, permet de restituer avec un maximum de vraisemblance l'emplacement du second des fûts supportant chaque arc : l'emploi de colonnes ou de cordons jumeaux est habituel dans ce type de décor, en général ${ }^{42}$, et il est attesté, dans le cas particulier de notre décorateur ${ }^{43}$, par plusieurs exemples indubitables. Il était tentant, une fois faites ces observations, de voir si la symétrie étendue à l'ensemble de la frise, qui avait aidé à restituer l'ordonnance des tessons du bol 2 de Glanım, donnait, cette fois, le moyen d'intégrer dans un ensemble cohérent tous les restes du gobelet.

J'ai donc entrepris une restitution graphique du décor. La hauteur de celui-ci est donnée, peut-être avec 1 ou $2 \mathrm{~mm}$ d'approximation, par l'établissement du profil. Il est donc pussible d'obtenir une projection circulaire de la frise, en partant du diamètre réel de sa base, el de disposer schématiquement les motifs selon la place qu'ils occupent sur les tessons. On peut voir ci-contre deux projections. L'une, celle du haut du décor (fig. 8. 2), s'est faite en plusieurs lemps : la mise en place des sujets conservés - réduits à leur schéma ou à leur axe vertical, espacés proportionnellement à leur distance réelle sur la surface du gobelet et orientés selon leur pente originelle - -. puis la restitution de l'emplacement des colonnes complémentaires, de l'autre còté des arceaux. Le déror ainsi composé couvre plus du tiers de la zone ornée et, s'il se répite régulièrement, ne peut, le faire qu'une fois ${ }^{44}$. J'ai donc, enfin, à litre d'essai, reporté par symétrie dans l'autre moitié du cercle les divisions déterminées en haut de la frise par les axes des colonnes et celui du double-fleuron, ce motif se trouvant, comme on l'a vu déjà, à égale distance des chapiteaux. L'aulre projection. celle du bas du décor (fig. 8, 1), a permis de situer précisément, par simple mesure de l'original, la position relative des socles des colonnes $(a$ et $b$ ) et celle de la grande feuille $(d)$ et d'orienter l'axe de chacun des trois motifs. Il est également loisible, à titre d'hypothèse, de fixer l'emplacement possible d'une troisième colonnette $\left(a^{\prime}\right)$ en le siluant en face d'une des bases conservées. En revanche, la pente de l'autre colonne (b) par rapport à la limite inférieure du décor implique un défaut d'exécution et ne permet aucune supposition valable quant à l'implantation symétrique du mème motif, d'autant moins que le pendant probable, de l'autre còté de la feuille, a disparu.

Il a suff, ensuite, de superposer les deux dessins et de les faire tourner l'un par rapport à l'autre autour du même centre, pour voir si la grille obtenue à partir du fragment supérieur rendait intelligibles les morceaux de la base de la frise : la place des deux colonnes, la présence, à leur gauche, tantôt d'une feuille, tantôt d'un chevron (c), la distance entre la feuille et la colonne se trouvaient-elles justifiées? On peut constater, d'après les schémas $A$ et $B$ (fig. 8, en bas), que deux positions relatives des figures partielles 1 et 2 , permettent d'intégrer sans aucune difficulté à un ensemble homogène tous les motifs conservés, en

41 A $1 \mathrm{~mm}$ près, la distance est la même entre l'axe du double-fleuron ol celui de chacun des deux chapilealux.

42 C. B., p. 230-231.

43 Bols de Glanum, Vintimille, Valence (deux fragments), de la coll. Gilles.

44 I.'hỵpothese, inverse, d'une irrégularité notable dans le partage de la surface interdit, en revanche, toute. supposition sur le nombre des répétitions possibles. On ne peut, évidemment, l'exclure, mais il faut cependant noter le soin apporté à l'exéculion du décor à métopes sur le bol le mieux conservé de la même série : celui de Glanum. 


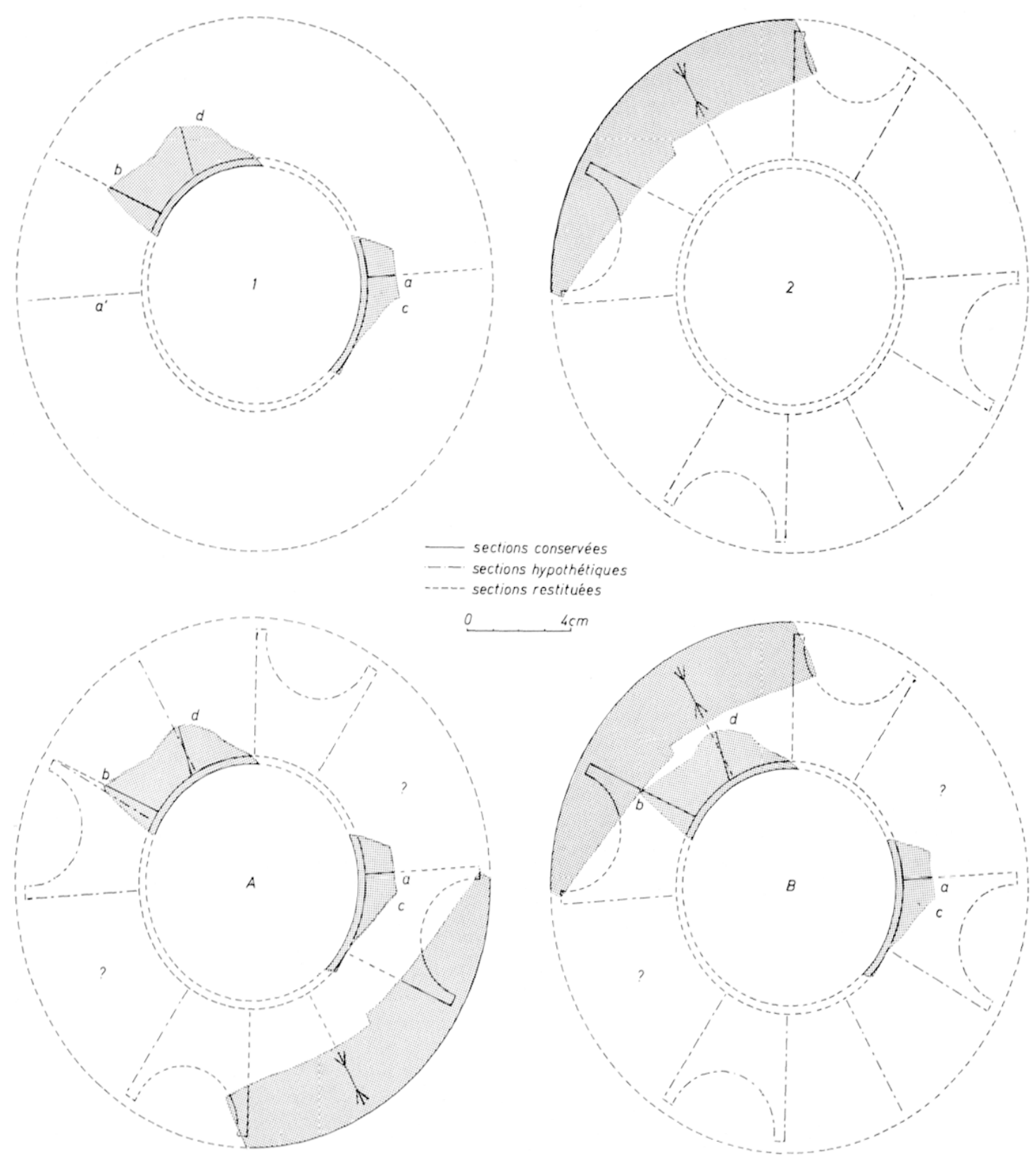

8 Schéma de la frise ornée. 1 : projection circulaire du bas du décor $l a$ et $b$ : axes des colonnes ; $c$ : place du fleuron; $d:$ axe de la feuille; 2 : projection du haul du décor (figuration schématique des principaux motifs et restitution hỵpothètique de leurs symét riques; ; $A$ et $B$ : hỵpothèses de restitution intégrant l'ensemble des fragments conservés.

rétablissant le tesson du haut du gobelet au-dessus de l'un ou de l'autre des décors de la base. Dans les deux cas, la place des deux socles s'explique par la restitution obligatoire d'une paire de supports pour chaque arceau, la feuille devient, au même titre que le doublefleuron, un ornement central de la large métope non coupée, tandis que le chevron est inclus dans un petit panneau, sous le feston. L'état du vase n'autorise pas à choisir l'une plutôt que l'autre des solutions. Tout au plus doit-on observer que, dans la première hypothèse $(A)$, aucun élément du décor n'est conservé à la fois par deux fragments et 
que deux défauts du mème genre (la pente incorrecte d'une colonne) sont attestés, l'un, par un tesson de la base (colonne b), l'autre, par le morceau supérieur (en face de b). Dans la seconde éventualité (B), en revanche, il semble que l'on ait deux morceaux contigus, ou, au moins, très voisins : la grande métope se trouve presque complètement meublée (la place et la dimension de la feuille expliquant la réduction en hauteur du double-fleuron), la colonne $b$ paraît se prolonger naturellement d'un fragment sur l'autre. 'Toutefois, l'inclinaison du motif, incontestable mais très faible dans le haut de la frise, ne me permet pas de garantir, sans risque d'erreur minime, l'exactitude de mon tracé.

Il paraît rraiment malaisé, en revanche, d'adopter dautres dispositions el de placer le panneau au double-fleuron ì un quart de cercle de la mélope il la feuille (a droile ou i gauche de celle-ci) : la différence de largeur entre les deux types de grands panneaux que suppose le schéma 1 obligerait a justifier la place de la feuille dans sa métope el l'implantation de deux colonnes (conservée et détruite) qui l'encadrent par une série d'asymétries ou d'irrégularités. ()r de tels défauls n'ont rien d'inconcevable dans un décor géométrique et l'on ne peut que les conslater. quand ils apparaissent ; on ne saurait, pour autant, les supposer gratuitement, surtout lorsqu'une aut re construction permel, dans l'état actuel du matériel, de rendre compte sans effort de tous les eléments conservés. Il est. sùr, en tout cas, que la paurrelé des vestiges sur lesquels se fonde celte restitution hypothétique. incite a marquer les limites de l'entreprise : il est impossible de rien imaginer pour les parties du décor entierement détruiles (fig. 8 : les vides, surtout les espares marqués d'un point d'interrogation); de plus, le déroupage symétrique de la surface n'implique pas, par principe, à lui seul, le retour identique du même poinģon décoralif dans la même case ${ }^{45}$. Il reste. cependant au lerme de l'expérience, que l'hypothese de travail : la répétition symétrique par rapporl au cenlre du décor d'une séquence de métopes connue seulement sur une face du vase, parait avoir arquis une plus grande vraisemblance. En effet. quelle que soil des solutions. celle que l'on retient, pour disposer le fragment mobile par rapport au pied du gobelet. on doit admettre que. si l'une des colonnes conservées en bas du décor appartient au groupe attesté par le haut de la frise, la place de la seconde se justifie nécessairement par l'existence, dans la partie détruite du vase, d'une construction semblable el dont la symétrie rend compte ${ }^{46}$.

Telle qu'on la pressent maintenant, l'ornementation de ce vase parait confirmer certains des caractères qu'avait précédemment légagés l'analyse d'autres fragments. Ainsi, rien dans l'économie du décor ne dément la sécheresse et la simplicité déjà observées à Glanum, Vintimille ou Valence et confirmée à Aix et Antibes : les motifs sont régulièrement répartis sur une surface cloisonnée aux moindres frais et toujours dépourvue de séparations horizontales. Ciependant, la pauveté du répertoire, ailleurs soulignée, ne doit pas ici nous abuser, compte tenu de l'importance des détériorations. Tout au plus peut-on remarquer que le décorateur reprend sans lassitude le même thème ornemental - lo jeu des colonnes et arceaux --- en associant les mêmes motifs. L'inventaire des poinçons s'enrichit peu, mais il est quand mème intéressant de noter un nourel emploi, sur un

45) Cf. l'exposi des systimes de composition que jai lire d'un ensimble d'observalions sur les vases du sud et du Centre (La composilion des décors géométriques à plan centré: comlribulion a l'slude du style des rases sigillés à mélopes, dans Rei Cretariae Romanae Faulorum, Arta XIV, p. 32-53.3.

46 Ia solution que je n’ai pas retenue implique, du fait de l'immulahilite des deux tessons solidajres du pied, que l'un des fragments ou les deux se trouvent hors du secteur couvert par le decor du lesson mobile. On devait. donc, même dans cotte hypothese, supposer l'existence d'une série de colommes apparemment symélrigue de celle que conserve le fragment supérieur. 

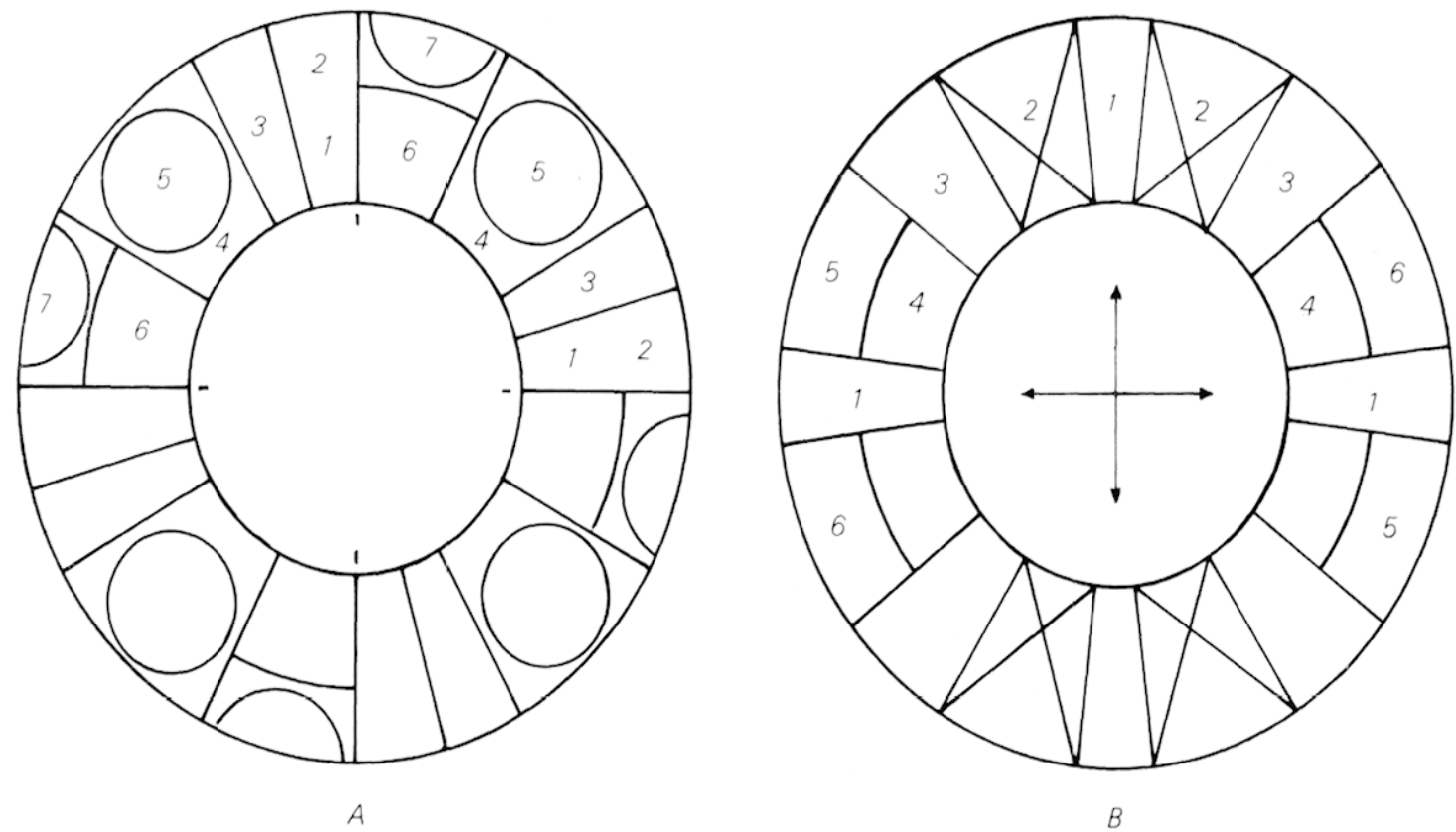

9 Exemples de structures complexes. $A$ : frise organiste en séquences répetés dans le même sens (d'après un vase

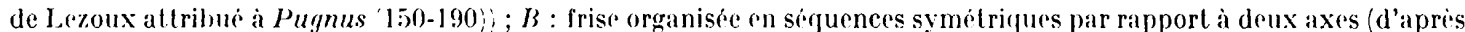
un vase anépigraphe de la Graufesenque :Faviens Cihaque chiffre désigne un des motifs qui se répètent d'un quart de vase a l'autre.

vase de composition, en apparence, largement géométrique, de la colonnette : la diversité des moyens employés dans les constructions régulières se trouve confirmée ainsi que les relations avec le répertoire du bol Gilles. Enfin, l'expérience tentée sur les tessons de Rognac, comme la restitution proposéc pour le vase de Glanum, tend à confirmer l'idée qu'on peut se faire de la technique d'organisation de ces frises : il parait de plus en plus vraisemblable que leur structure se fonde sur une symétrie par rapport à des axes, et le découpage régulier s'accompagne, chaque fois qu'une vérification est possible, de la répétition du même motif dans le même cadre. L'état du matériel interdit de contrôler la règle de distribution des poincons en toutes circonstances, mais rien, en tout cas, ne permet d'imaginer un autre mode d'organisation des surfaces à orner. J'ai montré ailleurs comment se répandait à Lezoux, surtout à partir de 130/140, l'habitude de composer le décor en séquences de plusieurs panneaux, non pas opposées deux à deux par rapport à des axes (fig. 9, B) (usage fréquent au $\mathrm{I}^{\mathrm{er}}$ siècle, en particulier à la (Graufesenque), mais se répétant dans le même ordre - le plus souvent quatre fois -.. et dans le même sens ${ }^{47}$ (fig. 9, A) et l'on pourra observer l'emploi du même système dans d'autres ateliers contemporains ${ }^{48}$. Or les exemples fournis par le décorateur aux colonnes, lorsqu'ils comportent

17 La composilion..., fig. 4, Iype $3 ; .4$ propos de CATVSSA, dans (jallia, 31, 1973, p. 99)-108.

48 Ce systeme se retrouve dans des ateliers en relations cotroites avec Iezoux : PATERNVS, aux .Iartres-deVeyre (J.-R. Terrisst, Les céramiques siqillées gallo-romaines des Marlres-de-Veyre (Pul-de-I)ôme), XIXe supp. a Gallia, 1968, pl. XVI, 480; CASVRIIS, à J.ubie (P. Karvitsch, Die Reliefsigillala vnn Ovilnu, Linz, I959, p. 204, pl. 63, 1 . Il ne paraît pas avoir connu ailleurs le même succès. 
MEPARTITIO DES POHNCON

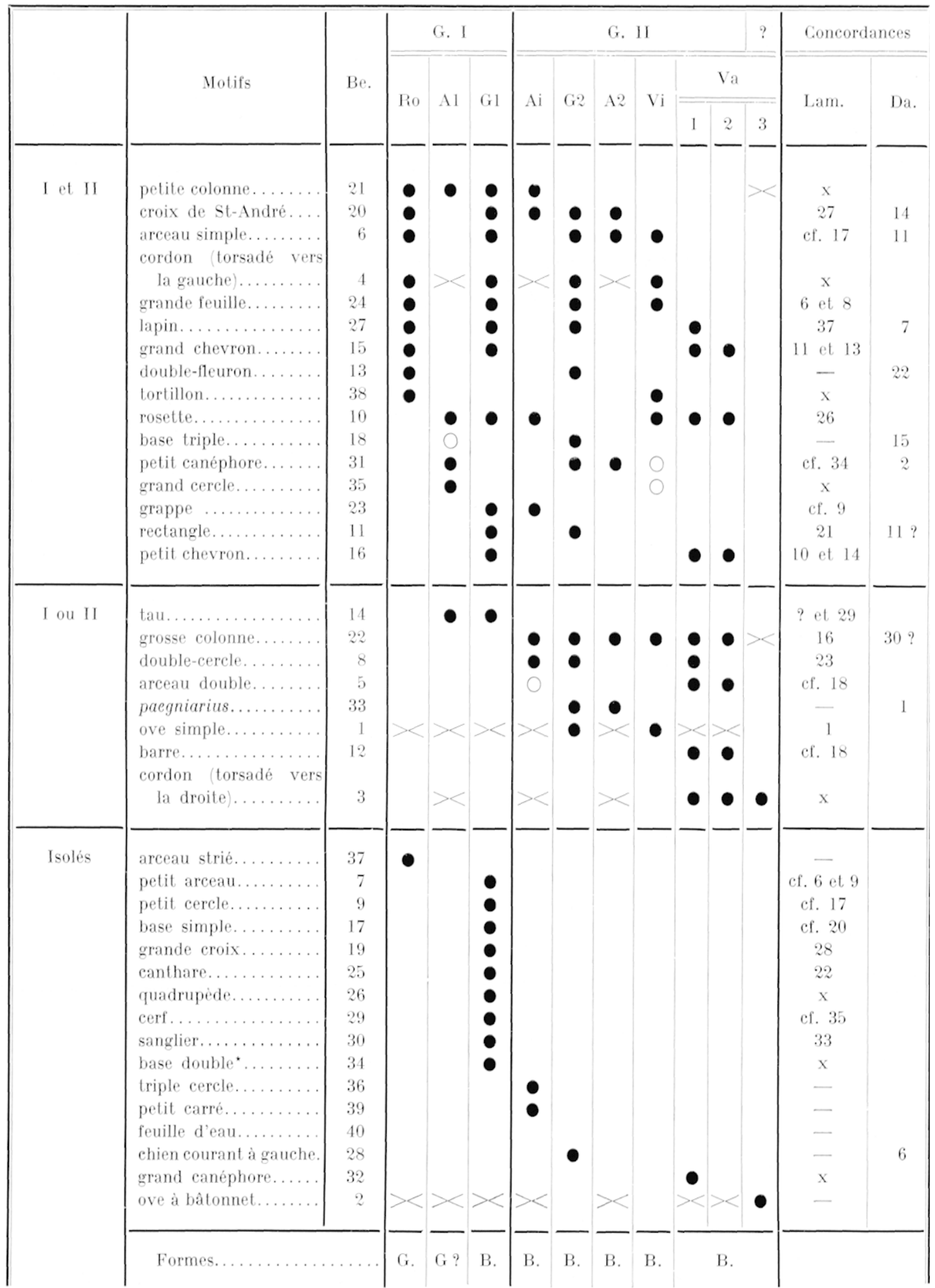


au moins une suite de plusieurs métopes différant soit par leurs dimensions, soit par leurs aménagements intérieurs, ne paraissent jamais, jusqu'à présent, impliquer l'enchaînement de groupes identiquement orientés ${ }^{49}$.

On aboutit ainsi à la définition d'un style composite : l'analyse du répertoire et de l'économie des métopes m’aviat amenée déjà à souligner des ressemblances entre l'atelier aux colonnettes et des fabriques du C'entre et de l'Est, datées, pour les plus anciennes, du deuxième tiers du ${ }_{1 \mathrm{I}}{ }^{\mathrm{e}}$ siècle, et de dégager certaines des originalités de notre potier. Cielui-ci, en effet, mélange les procédés et les motifs, ailleurs distincts, des décors à arcades et des frises à métopes coupées, pour obtenir des compositions plus économiques et plus sèches encore que celles de la fin du II $^{\mathrm{e}}$ siècle à Lezoux, tout en observant, dans la plupart des cas, les mèmes principes de régularités ${ }^{50}$. L'examen du gobelet et sa confrontation avec d'autres pièces du même artisan font ressortir une autre particularité de cette technique : la construction de certaines frises selon des règles de symétrie par rapport à des axes. L'etat des fragments ne permet, malheureusement, pas plus que le nombre des exemples de caractériser plus exactement ces compositions, mais l'apparente diversité des découpages paraît, actuellement, plus proche de la variété des modèles du sud de la Gaule, que de la

49 Le bol de Vintimille ot Antibrs 1, dans leur état actuel, conservent deux types de métopes alternés. Le premier fragment de Valence comporte un panneau étroit encadré par deux larges métopes de contenu identique; le deuxième présente une partie du large panneau et un petit morceau d'un troisième genre de métope orné differemment des deux autres et de dimensions inconnues. I.e premier lesson est compatible avec une construction allerné fondée sur deux panneaux, ou avec une structure symétrique par rapport à un axe. Si les deux fragrnents apparliennent à un même bol, rien ne prouve que la succession des lrois métopes différentes soit répété dans le même sens. l.e vase d'Aix, enfin, avec ses trois panneaux dissemblables, ne permet pas de choisir ent re une disposition symetrique par rapport à des axes et la répétition de séquences identiquement orientées.

5) (: B., P. 226-232.

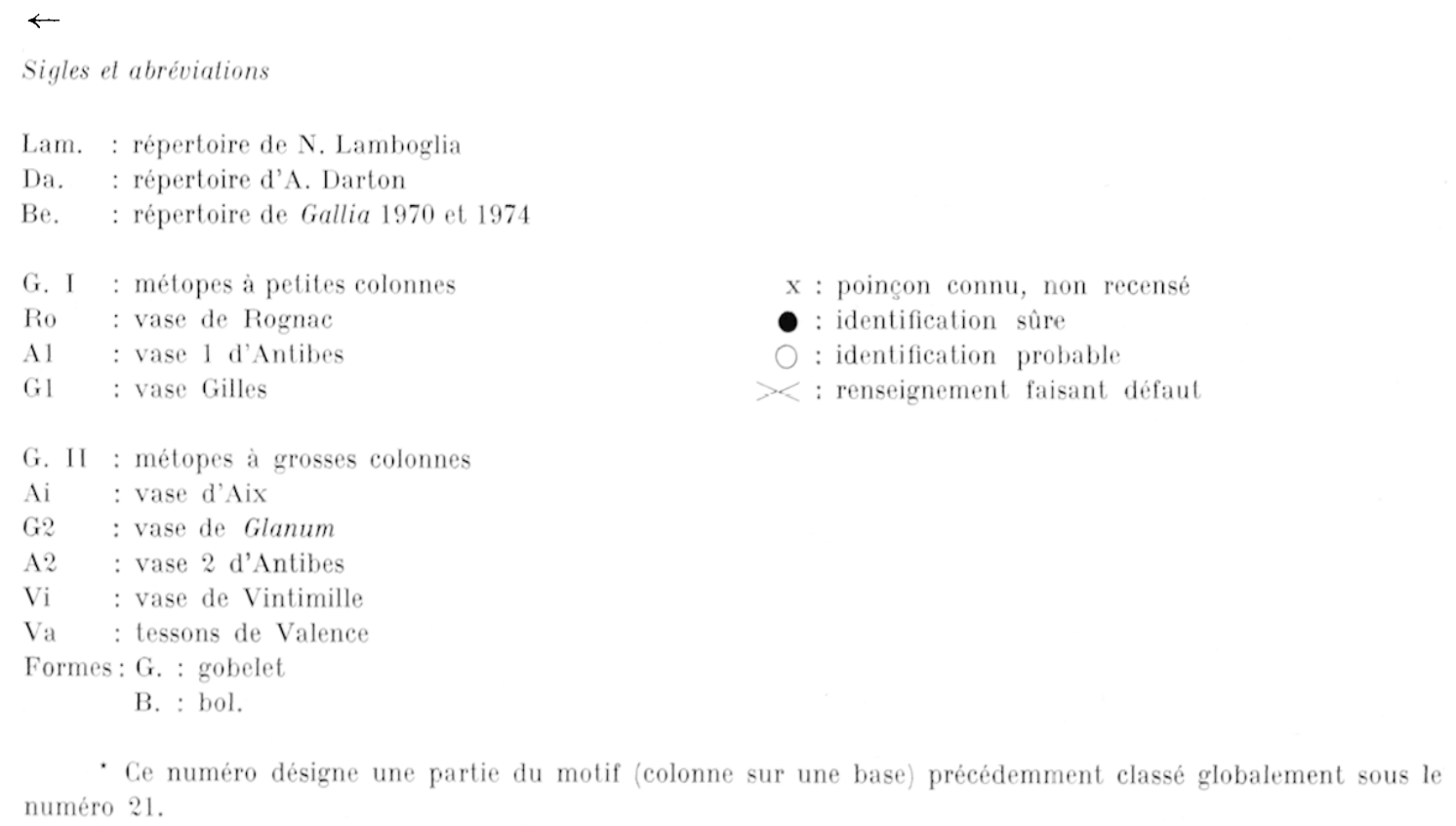


similitude figée des structures symétriques conservées à la fin du $\mathrm{II}^{\mathrm{e}}$ siècle dans le lientre et dans l'Est ${ }^{51}$.

Le gobelet de Rognar parait ainsi résumer des influences diverses : sa forme, aussi hien que son décor rérèlent un mélange de caractères tardifs (galbe, séeheresse du style) et d'archaïsmes ou de survivances (technologie, structure du decor), sans qu'on sache, pour les derniers de ces caractères. s'il s'agit d'imitations délibérés ou de rencontres fortuites. Hors de toute stratigraphie déterminante il est difficile de dater l'objet avec précision. Toutefois, les analogies typologiques -... qui fournissent le lerminus post quem le plus bas... incitent à le placer, au plus tòt au ur $^{\mathrm{e}}$ siècle. Accepter cette datation "haute" oblige à supposer des relations précoces entre la sigillée claire $\mathrm{B}$ et des produits de l'Argonne fabriques avant les invasions de 276 (puisque ces rlernières semblent avoir interrompu pendant une durée mal déterminée le travail des ateliers de l'Est ${ }^{52}$. Dater le vase du rve siècle n'implique pas obligatoirement l'inlluence exclusive des gobelets à pied fermé (forme 33.5) de la seconde périorle de l'Argonne, mais a d'autres conséquences : la date de la destruction des bâtiments de Rognac se trouve abaissée et la présence de sigillée claire B décorée, attestée tardivement dans le delta du Rhòne. De plus, l'existence de cette pièce pose alors nettement un probleme aux céramologues : celui de la durée de la fabrication des vases moulés décorés de colonnes. J'ai souligné à plusieurs reprises l'homogénéité du répertoire du décorateur des moules et mis an evidence également la qualité presque uniforme de la technique de construction des frises s. $^{53}$ Le tableau ci-joint permet, d'ailleurs, de mesurer la ventilation des poincons que j'ai contròlés directement ou indirectement ${ }^{54}$ et de constater que la différence la plus tangibles5 oppose un vase 1). 37 : le bol Cilles, au reste des produits connus. Le tableau montre, en effet, dans sa troisieme section, le nombre considerable des poincons propres à ce décor. Cependant cette particularité s'est

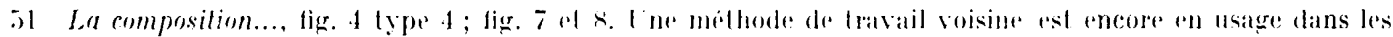

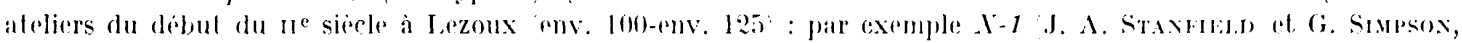

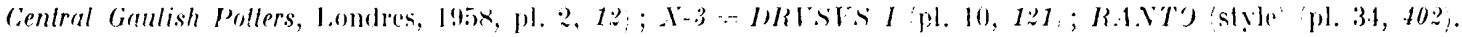
Mais cefle construction lend a se figer systematiquement en une sorle de composition en eloile : lat frise est divisere,

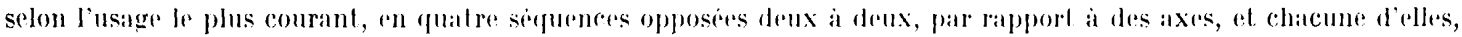

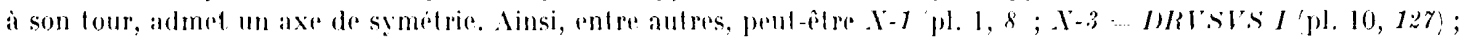

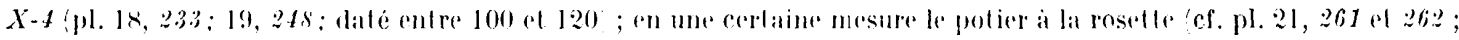

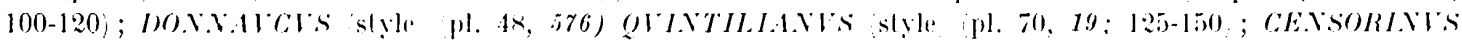

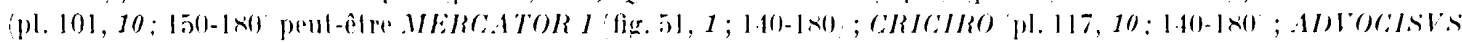

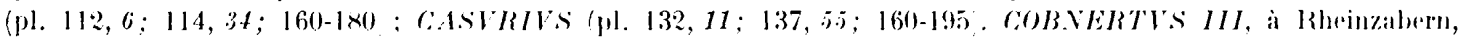
procide da la mème façon H. liskis. Katalog VI, Tafelhand. Die Bilderschüsscln der romischen Töpfer von Rheinza-

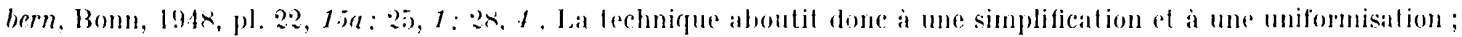
d'ailleurs, dans de nombreux cas, a loboux el, surtout, dans les ateliers de l'bst, la division den sioguences complexes

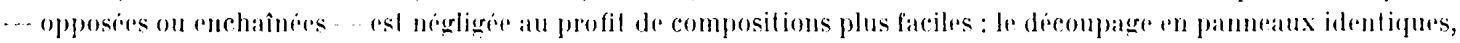
ou l'alternance, une ou plusieurs fois répétée, de deux métopes inegales enlee elles.

\$2 lif. supra, 11. 30 .

53 (:.B., p. 230-232 et supra p. $86-\times 9$ et $98-101$.

it I.̈identification des poincons de Vintimille, par ailleurs atlestes, s'est faild par analogite.

5i) Les distinctions que jai failes dans le lableau, pour plus de clarte, d'apres lat mature des separalions pelites ou grosses colommes of les formes bols ou gobelets. ne permettent pas faute de confirmalions exterienres rel d'un nombre suflisant d'exemples, dobserver des groupements convaineants par variations concomilantes. Itaul re part, la taille du grobelet semble impliquer surtout un rapport privilegio rentre les colommelles a simples bases et les pelits moules la hauleur de la frise dimimuant arec lo diametre tandis que la forme hemispherique de la partie moulee ne laisse pas projuger de l'usage, uniforme ou divers, qui elail fait de la matrice. 
déjà atténuée du fait de la connaissance de nouveaux tessons (première section, 21, 23; deuxième, 14) et l'on ne peut écarter l'idée que les dimensions exiguës de la plupart des fragments - alors que le vase Gilles nous restitue une large surface décorée - sont pour une part appréciable responsables d'une telle disparate. En revinche, la structure du décor, l'importance - minime par rapport à la longueur tolale de la frise - des compositions géométriques et le caractère bâtard de celles-ci constituent, à mon avis, la singularité la plus notable de ce bol. Tous les autres décors, en revanche, y compris celui du gobelet, paraissent appartenir à la mème famille. Il ne semble donc pas possible de confirmer par ce moyen le caractère supposce plus tardif du vase de Rognac.

Par ailleurs, l'examen des échantillons - c'est-à-dire des produits finis et non plus de la composition du décor, nécessairement liée à la date de fabrication du moule - ne permet pas davantage de constituer des groupes sùrement distincts : ni la qualité du relief (déterminée en l'occurrence par les dégradations postéricures à lia fabrication), ni l'aspect et la dureté de la pâte et de l'engobe - il y a, ou peu s'en faut, autant de nuances que de vases $^{56}$ - ne témoignent effectivement d'autre chose que d'une maitrise incertaine, ou variable, des phénomènes de cuisson ${ }^{57}$. En tout cas, le gobelet de Rognac se situe, de ce point de vue, parmi les exemples les plus conformes à la définition de la sigillée claire B donnée par N. Lamboglia ${ }^{58}$.

La question consiste donc à se demander, dans l'hypothèse d'une fabrication tardive du gobelet et en l'absence de critères chronologiques intrinsèques ou extrinsèques contraignants, si la fabrication des vases moulés à colonnettes s'est prolongée continuement de 250 environ - date extrème admise par N. Lamboglia pour le niveau qui correspond à la construction du théâtre de Vintimille ${ }^{59}$ et dans lequel se trouve le seul vase découvert dans une suite stratigraphique homogène -- jusqu'à un moment imprécis lu rve siècle, ou si les produits issus de ces moules ont été fabriqués de façon discontinue, mais peut-être avec les mêmes matrices, d'abord vers le milieu du III siècle, puis au Ive. Je n'ai ni l'intention, ni la possibilité de résoulle ce problème et je me bornerai à apporter au débat quelques observations.

La fabrication prolongée ou attardée de poteries moulées ressortissint à la sigillée claire B n'a théoriquement rien d'absolument inconcevable, à partir du moment où l'on admet ... pour des raisons typologiques, en particulier 60 ... la production de vases lisses de cette qualité jusqu'au rve siècle. Toutefois, la possibilité d'une création continue de vases décorés de colonnettes durant plus de cinquante ans, ou la reprise d'une technique et le remploi d'un outillage après plusieurs décennies d'interruption paraissent peu proba-

56 La pate varie du beige plus ou moins pale !hol 2 de Glanum, gobelet, al beige rosé, pour des exemplaires

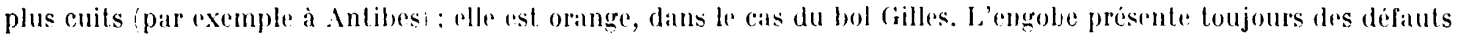
d'adherence, au moins à l'exterirur on a l'interieur et sa couleur va du rouge brique foncé (Aix, Gilanum à l'orange vif (Valence, bol Gille's, Antibes, hognac.

57 Les examens physico-chimiques, qui n'ont pu être fait encore que sur une partie de ces fragments, n'ont apporle que la confirmation de cette apparente diversite.

58 O.c., 1958 , р. 297 .

$59 \quad$ O.c., 1962, p. 275.

60 X. Lambogin, o. c., I958, р. 330 ; J. Rigorr, Les sigillies puliorhritiennes grises el orangées, dans Gallia, X.XVI, 1966, p. 189-190; 1. DarTox, o. c., p. 150-151. 
bles : on comprend mal, en effet, qu'un atelier, si modeste fût-il, ait produit si peu pendant un tel laps de temps; on ne voit pas beaucoup mieux pourquoi une fabrication de si médiocre importance aurait pu reprendre après deux générations d'oubli. Aussi, compte tenu de l'inventaire actuel du matériel, nécessairement incomplet, mais aussi de l'énorme disproportion entre le nombre des vases lisses de même qualité et celui des échantillons décorés de colonnes connus de nous, inclinerait-on raisonnablement à penser que cette production a pu se renfermer en quelques décennies. L'ignorance où nous nous trouvons en ce qui concerne la localisation des ateliers complique, il est vrai, l'interprétation des faits : la distance entre le centre de diffusion et les points de découverte des vases pourrait expliquer en partie la rareté du matériel et son apparente disparité typologique et chronologique. Encore faudrait-il que des trouvailles plus nombreuses ailleurs vinsent à l'appui de cette hypothèse. Ce n'est, actuellement, pas le cas : l'idée d'une fabrication limitéc dans le temps paraît donc, provisoirement, la plus vraisemblable. Quant à la date probable de la production, force est de noter la précarité des indices archéologiques dont nous disposons en faveur d'une chronologie basse : les fragments de Saint-Rémy, de Valence, d'Aix et Antibes 1 n'ayant pas de contexte connu ou sûr, restent les tessons de Vaison, Antibes 2 et le vase de Vintimille. Le bol de Vaison provient de fouilles faites en 1961 dans un niveau de réoccupation des $\mathrm{III}^{\mathrm{e}}$ et $\mathrm{IV}^{\mathrm{e}}$ siècles : il n'est pas possible, faute de plus grandes précisions, de dater plus étroitement la destruction du vase ni, a fortiori, sa fabrication ${ }^{61}$. De même Antibes 2 a bien été trouvé avec des minimi attribués à la période 330-360, mais la variété du reste du matériel et le remaniement du terrain interdisent de faire obligatoirement des tessons les contemporains des monnaies ${ }^{62}$. Nous revenons donc au vase de Vintimille et à sa chronologic haute ${ }^{63}$.

On peut envisager, enfin, l'éventualité d'une fabrication tardive "accidentelle " du gobelet. Nous avons vu, plus haut que les similitudes typologiques sur lesquelles on pouvait s'appuyer ne sont pas assez rigoureuses pour imposer un modèle du ive plutôt que du ${ }_{\text {III }}{ }^{\mathrm{e}}$ siècle. Quant à faire fond sur le caractère isolé de cette pièce, la prudence est, à mon avis, de rigueur. Il est difficile d'assurer que le gobelet représente un lype nouveau, c'est-àdire un ensemble de caractères associés de façon régulière dans la forme d'une série d'objets, mais il semble déjà qu'il subsiste des fragments de vases comparables à celui-ci par l'une ou l'autre de leurs parties. Je n'ai pu, faute d'informations suffisantes, contrôler les tessons qui, selon F. Pallarès ${ }^{64}$, conservent le profil du bas du gobelet, ni m'assurer de la nature de leur décor. I'autre part, le fragment de piédestal de Valence ${ }^{65}$ est trop exigu pour se prêter

61 C. Informalions de Callia, XX, 1962, p. 683-684 (la numerotation du tesson date la campagne de fonilles . Ni $\Lambda$. Dumoulin, ni B. L.iou, aupres de qui je me suis informée, n'ont pu sur ce point me fournir de renseignements plus complets.

62 Je dois ces informations à l'amabilité de G. B. Kogers.

63 C'est-a-dire entre 200 el 250. Même la chronologrie la plus basse, que rejelle X. Lamboglia et qui se fonde sur la découverte, dans une fouille ancienne, d'une monnaie de Telricus, $270-273$, ne permettrait guere de descendre bien au-delà du troisième quart du un $^{\mathrm{e}}$ siecle.

64 Ces renseignements m'ont été fournis oralement par Mlle Pallaris, qui, sur le champ, n'a pu me donner les références exacles de ces pieces.

65 Le lesson ma été signalé par $A$. Blanc. Aucun des nombreux fouilleurs ou conservaleurs que j'ai consultés tant en Provence que dans le Ianguedoc-Ihoussillon ne m'a indiqué d'autres pièces se prètant à comparaison. 
à une comparaison satisfaisante. Ces vestiges, du moins, permettent de soupçonner l'existence d'un groupe encore mal connu. Et ces présomptions peuvent être renforcées par un des échantillons d'Antibes: Antibes 1, en effet, est marqué, à l'intérieur, de traces accentuées de travail au tour et la qualité assez soignée de la fabrication rend peu vraisemblable la négligence d'un tourneur de bols; en revanche, ces caractéristiques conviennent à une panse d'oroïde ${ }^{66}$.

Le gobelet de Rognac apparait donc, par sa technologie et son décor, comme une variante tardive des gobelets ou ovoüdes moulés, produits, au $\mathrm{I}^{\mathrm{er}}$ siècle, dans le Sud et, au II ${ }^{\mathrm{e}}$, surtout à Lezoux. Des particularités morphologiques trahissent une parenté avec certains vases d'Argonne ${ }^{67}$ et l'on pourrait hésiter entre l'hypothèse d'une fabrication prolongée d'une série pourtant peu représentée et celle de relalions entre la sigillée claire B et les produits de l'Argonne plus précores que ne le proposait X. Lamboglia ${ }^{68}$. La seconde de ces suppositions parait, dans l'état actuel des connaissances, la plus raisonnable, mais il y a lieu d'espérer, compte tenu des quelques indices déjà signalés, que l'identification ou la découverte d'autres témoins comparables et l'étude de leur environnement archéologique permettront de mieux connaître de nouveaux rameaux de cette famille céramique et de résoudre de façon irréfutable le problème aujourd'hui posé.

\section{Colette Bŕmont.}

66 Le galbe, autant qu'on en puisse juger, est comparable à celui d'un bol, mais le gobelet de Rognac présente la même particularité, au point qu on peut se demander si les mêmes moules n'ont piss servi - de façon normale ou exceptionnelle - à produire les deux types de vases.

67 Je n'ai trouvé jusqu'à prèsent aucune autre catégorie de vases qui se prêtả à la même comparaison.

68 Ia date et les modalités des relations entre l'Argonne el les aleliers, supposés Rhodaniens, qui produisent la sigillée claire B restent à préciser. En tout cas, il me paraît utile de souligner qu'il n'est pas obligratoire d'expliquer des ressemblances par la seule diffusion des modeles presumés el l'existence de courants commerciaux réguliers : les hommes aussi voyagent of jl suffit que le fabricant ait connu les formes dont il paraît s'inspirer. Par ailleurs on notera que la grande ressemblance entre certains vases lisses des deux regions, dates du ni siecle a déjà eté remarcjuée (A. DARTON, o. c., p. 142). 